\title{
UK Renal Registry 20th Annual Report: Chapter 2 UK Renal Replacement Therapy Adult Prevalence in 2016: National and Centre-specific Analyses
}

\author{
Stephanie J MacNeill ${ }^{\mathrm{a}}$, Daniel Ford ${ }^{\mathrm{b}}$, Katharine Evans ${ }^{\mathrm{a}}$, James F Medcalf ${ }^{\mathrm{c}}$ \\ ${ }^{a}$ UK Renal Registry, Bristol, UK; ${ }^{b}$ University Hospital Coventry and Warwickshire, Coventry, UK; \\ 'University Hospitals of Leicester NHS Trust, Leicester, UK
}

\section{Keywords}

Chronic kidney disease . Clinical Commissioning Group . Comorbidity · Diabetes - Dialysis · End stage renal disease · Established renal failure - Ethnicity $\cdot$ Haemodialysis · Peritoneal dialysis . Prevalence - Renal replacement therapy • Transplantation · Treatment modality

\section{Summary}

- There were 63,162 adult patients receiving renal replacement therapy (RRT) in the UK on 31 December 2016, an absolute increase of 3.1\% from 2015.

- The actual number of patients increased by $0.9 \%$ for haemodialysis (HD), 5.1\% for those with a functioning transplant and less than $0.1 \%$ for peritoneal dialysis (PD).

- The UK adult prevalence of RRT was 962 per million population ( $\mathrm{pmp}$ ). The reported prevalence in 2000 was 523 pmp.
- The number of patients receiving home HD increased slightly from 1,175 patients in 2015 to 1,256 patients in 2016 .

- In 2016 the median age of prevalent patients was 59 years (HD 67 years, PD 64 years, transplant 54 years). In 2000 the median age was 55 years (HD 63 years, PD 58 years, transplant 48 years). The percentage of RRT patients aged greater than 75 years in 2016 was $16.0 \%$.

- For all ages, RRT prevalence in men exceeded that in women, peaking in age group 80-84 years at 3,072 pmp in men and in the 70-74 years age group at 1,657 pmp in women.

- The most common identifiable renal diagnosis was glomerulonephritis (19\%), followed by diabetes $(17 \%)$, other $(17 \%)$ and aetiology uncertain (15\%).

- Transplantation continued as the most common treatment modality (54\%), HD was used in $40 \%$ and PD in $6 \%$ of RRT patients.

\section{KARGER}

Fax +4161306 1234 E-Mail karger@karger.com www.karger.com/nef
This article is licensed under the Creative Commons AttributionNonCommercial-NoDerivatives 4.0 International License (CC BYNC-ND) (http://www.karger.com/Services/OpenAccessLicense) Usage and distribution for commercial purposes as well as any
distribution of modified material requires written permission.
Stephanie MacNeill

UK Renal Registry, Southmead Hospital, Southmead Road, Bristol, BS10 5NB, UK

Email: renalregistry@renalregistry.nhs.uk 


\section{Introduction}

This chapter presents data on all adult patients on RRT in the UK at the end of 2016. The UK Renal Registry (UKRR) received data returns for 2016 from all five renal centres in Wales, all five in Northern Ireland and 51 in England. Cambridge renal centre (Addenbrooke's) was unable to submit 2015 or 2016 data at patient level prior to the UKRR closing the database and only provided summary numbers of patients starting RRT by treatment modality. This centre is therefore excluded from most analyses in this chapter. Data from all nine centres in Scotland were obtained from the Scottish Renal Registry. Demographic data on children and young adults can be found in chapter 4 .

These analyses of prevalent RRT patients are performed annually to aid clinicians and policy makers in planning future RRT requirements in the UK. It is important to understand national, regional and centre level variation in numbers of prevalent patients as part of the capacity planning process. In addition, knowledge about variation in case mix is also reported to improve understanding of where resources should be focussed to improve equity of provision of RRT in the UK.

The term established renal failure (ERF) used within this chapter is synonymous with the terms end stage renal failure and end stage renal disease, which are in more widespread international usage. Patients have disliked the term 'end stage' which reflects the inevitable outcome of this disease.

\section{Methods}

Crude prevalence ratios were calculated pmp and age/sex standardised prevalence ratios were calculated as detailed in appendix D: Methodology used for Analyses of Clinical Commis- sioning Group (CCG)/Health Board (HB) Incidence and Prevalence Rates and of Standardised Ratios (www.renalreg.org).

Throughout this chapter, HD refers to all modes of HD treatment, including haemodiafiltration (HDF). Several centres reported significant numbers of patients on HDF, but other centres did not differentiate this treatment type in their UKRR returns. Where joint care of renal transplant recipients between the referring centre and the transplant centre occurred, the patient was usually allocated to the referring centre (see appendix B2 for the allocation procedure). Thus the number of patients allocated to a transplant centre is often lower than that recorded by the centre itself and conversely, pre-emptively transplanted patients were sometimes allocated to the transplanting centre rather than the referring centre if no transfer out code had been sent through. Queries and updated information are welcomed by the UKRR at any point during the year if this has occurred.

Prevalent patients on RRT in 2016 were examined by time on RRT, age group, sex, ethnic origin, primary renal disease, presence of diabetes and treatment modality (see appendix H: Coding, www.renalreg.org). In the analysis of prevalence, only adult patients on RRT contributed to the numerator and denominator.

Time on RRT was defined as median time on treatment and was calculated from the most recent start date. Patients without an accurate start date were excluded from this calculation.

Analyses were done for the UK as a whole, by UK country, at centre level and split by treatment modality when appropriate.

Chi-squared test, Fisher's exact test, linear regression and Kruskal Wallis tests were used as appropriate to test for significant differences between groups. The data were analysed using SAS 9.3.

\section{Results}

Prevalent patient numbers and changes in prevalence

The number of patients for each country (table 2.1) was calculated by totalling the number of patients in each renal centre located in the country. These numbers differ marginally from those quoted elsewhere in this report when patients are allocated to geographical areas by their individual post codes, because some centres treat patients from across national boundaries.

Table 2.1. Prevalence of adult RRT in the UK on $31 / 12 / 2016$

\begin{tabular}{|c|c|c|c|c|c|}
\hline & England & N Ireland & Scotland & Wales & UK \\
\hline Total estimated population, mid-2016 (millions)* & 55.3 & 1.9 & 5.4 & 3.1 & 65.6 \\
\hline Prevalence ratios PD (pmp) & 56 & 41 & 42 & 66 & 55 \\
\hline Prevalence ratios dialysis (pmp) & 446 & 382 & 395 & 439 & 440 \\
\hline Prevalence ratios transplant (pmp) & 519 & 574 & 522 & 545 & 522 \\
\hline
\end{tabular}

${ }^{*}$ Data from the Office for National Statistics, National Records of Scotland and the Northern Ireland Statistics and Research Agency - based on the 2011 census

Pmp - per million population 
There were 63,162 adult patients receiving RRT in the UK at the end of 2016, giving an adult UK population prevalence of $962 \mathrm{pmp}$ (table 2.1) compared with $941 \mathrm{pmp}$ in 2015. RRT prevalence increased in all UK countries in 2016. Since 2015 the prevalence of dialysis in the UK remained steady at $440 \mathrm{pmp}$ and there were increases in the prevalence of transplant from $501 \mathrm{pmp}$ in 2015 to 522 in 2016. There had been a slow decline in PD prevalence in previous years, but prevalence in 2016 remained at the same level as in 2015. As observed in the previous year, Northern Ireland exhibited a higher RRT prevalence for patients aged 75 years and older compared with the other UK countries (figure 2.1). In the UK, RRT prevalence in patients aged $80-84$ continued to rise from 2,044 per million age related population (pmarp) in 2015 to 2,098 pmarp in 2016 and in patients aged $\geqslant 85$ years from 1,084 pmarp in 2015 to 1,129 pmarp in 2016. This trend has been remarked upon over a number of years and the observed aging of the prevalent population is likely due in part to improving patient survival.

\section{Prevalent patients by RRT modality and centre}

There was a marked variation in the number of prevalent patients across renal centres and the distribution of their treatment modalities also varied widely (table 2.2 ).

\section{Changes in prevalence}

The prevalent UK RRT population grew by 3.2\% between 2015 and 2016 (table 2.3), an annual growth rate which has been fairly consistent over the last 10-15 years (figure 2.2).

The increase in prevalence was greatest in Northern Ireland (4.6\%) and most modest in Wales (0.9\%).

The number of prevalent HD patients increased by $0.1 \%$ in 2016 compared with 2015 , which was a much smaller increase than that seen between 2014 and 2015 (2.7\% growth in prevalence pmp). There continued to be an increase in prevalent transplant patients (4.2\% pmp) and very little change in prevalent PD patients (0.6\% pmp decrease).

The average annual change in prevalent patients between 2012 and 2016 was a 1.0\% pmp increase in $\mathrm{HD}, 2.1 \% \mathrm{pmp}$ fall in PD and 4.6\% pmp growth in prevalent transplant patients (table 2.4). In the same period there was an average annual $15.5 \%$ pmp growth (an absolute increase of 451 from 737 to 1,188 ) in the use of home haemodialysis (data not shown).

The long-term (1997-2016) UK prevalence pattern by treatment modality is shown in figure 2.2. The steady growth in transplant numbers was maintained in 2016.

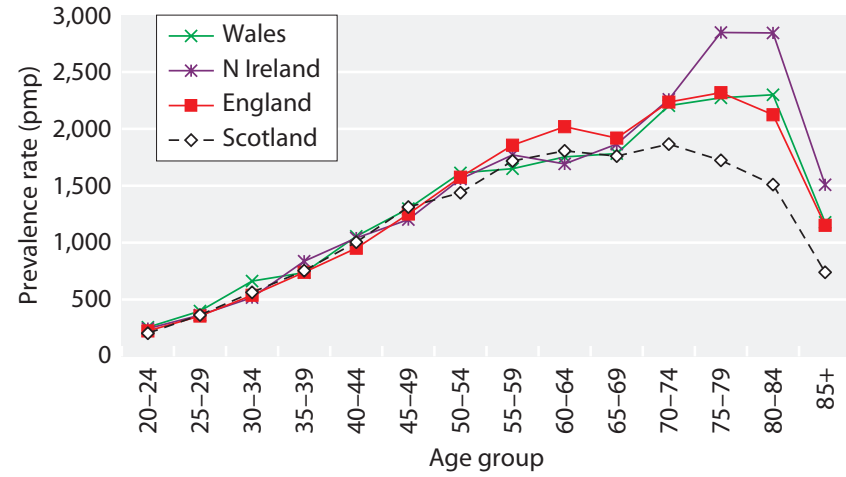

Fig. 2.1. RRT prevalence pmp by age group and UK country on $31 / 12 / 2016$

The increase in home haemodialysis patient numbers over this period has been associated with more than a doubling in prevalence, from $1.9 \%$ of the dialysis population in $2006(N=445)$ to $4.4 \%$ in $2016(N=1,256)$. In contrast, PD has fallen by $4.9 \%$ between 2006 and 2016.

Prevalence of RRT in Clinical Commissioning Groups in England (CCGs), Health and Social Care Areas in

Northern Ireland (HBs), Local Health Boards in

Wales (HBs) and Health Boards in Scotland (HBs)

The need for RRT depends upon many factors such as primary renal diagnosis, but also on social and demographic factors such as age, sex, social deprivation and ethnicity. Hence, comparison of crude prevalence ratios by geographical area can be misleading. This section, as in previous reports, uses age and sex standardisation to compare RRT prevalence. The ethnic minority profile is also provided to help understand the differences in standardised prevalence ratios (SPRs).

There were substantial variations in the crude CCG/ HB prevalence ratios pmp (table 2.5), from $639 \mathrm{pmp}$ in Lincolnshire (NHS South West Lincolnshire, population $125,200)$ and $641 \mathrm{pmp}$ in Orkney (Orkney, population 21,900 ) to $1,773 \mathrm{pmp}$ in Brent (NHS Brent, population $328,300)$. However, as described in table 2.5, estimates for some CCGs (denoted with an ${ }^{\mathrm{a}, \mathrm{b}}$ in table 2.5 , including NHS South West Lincolnshire) may be underestimated given that $5-15 \%$ of patients from these CCGs were estimated to be treated at the Cambridge renal centre, which was unable to provide patient-level data in 2015 or 2016.

There were similar variations in the SPRs (ratio of observed: expected prevalence given the age/sex breakdown of the CCG/HB) from 0.57 (Orkney) to 2.37 (NHS Bradford City) (table 2.5). Confidence intervals are not presented for the crude ratios pmp for 2016 but 
Table 2.2. Number of prevalent RRT patients by treatment modality and centre on $31 / 12 / 2016$

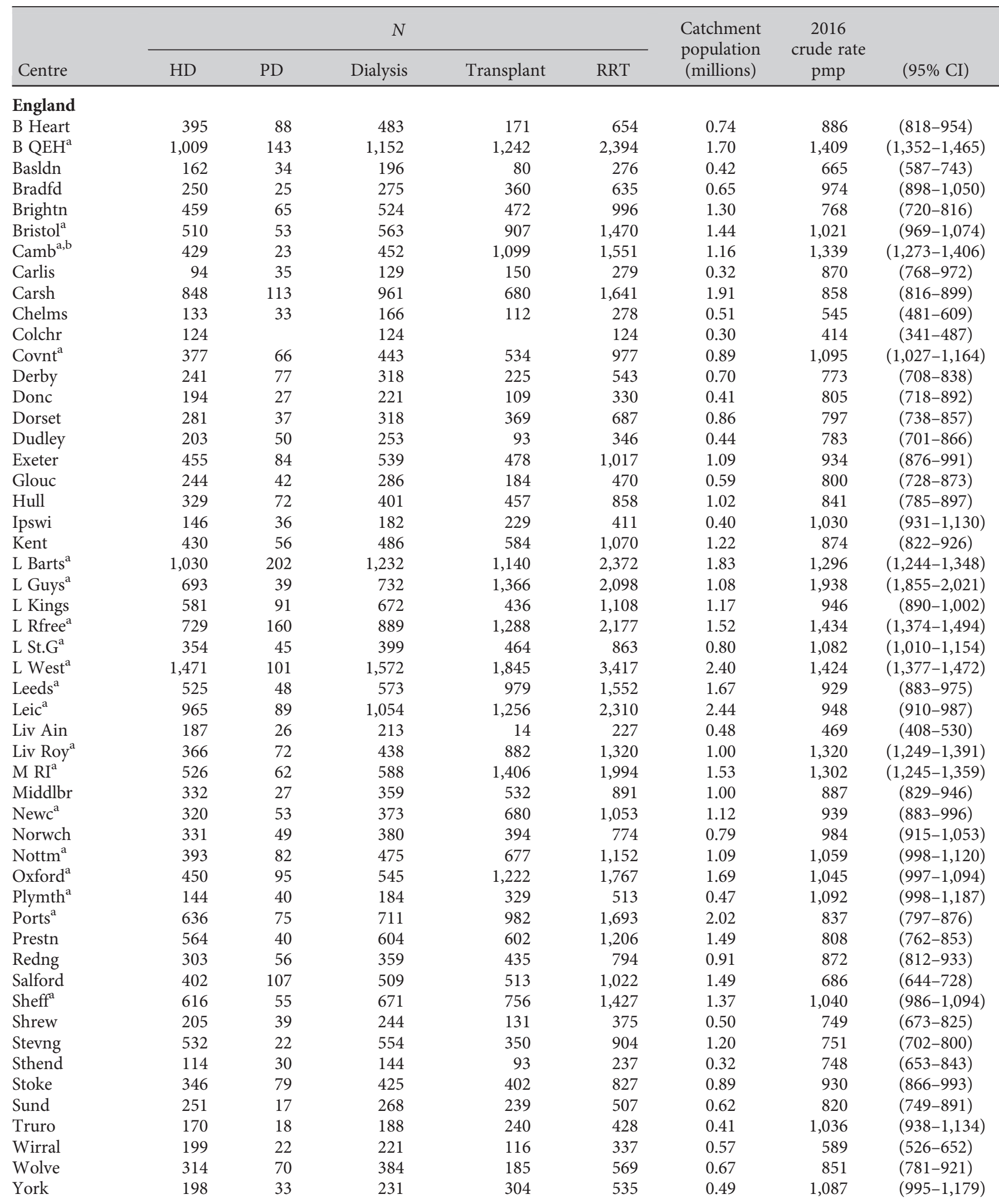


Table 2.2. Continued

\begin{tabular}{|c|c|c|c|c|c|c|c|c|}
\hline \multirow[b]{2}{*}{ Centre } & \multicolumn{5}{|c|}{$N$} & \multirow{2}{*}{$\begin{array}{l}\text { Catchment } \\
\text { population } \\
\text { (millions) }\end{array}$} & \multirow{2}{*}{$\begin{array}{l}2016 \\
\text { crude rate } \\
\text { pmp }\end{array}$} & \multirow[b]{2}{*}{$(95 \% \mathrm{CI})$} \\
\hline & $\mathrm{HD}$ & $\mathrm{PD}$ & Dialysis & Transplant & RRT & & & \\
\hline \multicolumn{9}{|c|}{ Northern Ireland } \\
\hline Antrim & 123 & 16 & 139 & 102 & 241 & 0.29 & 818 & (714-921) \\
\hline Belfast $^{\mathrm{a}}$ & 194 & 24 & 218 & 611 & 829 & 0.64 & 1,302 & $(1,213-1,390)$ \\
\hline Newry & 87 & 21 & 108 & 129 & 237 & 0.26 & 907 & $(792-1,023)$ \\
\hline Ulster & 102 & 6 & 108 & 58 & 166 & 0.27 & 624 & $(529-719)$ \\
\hline West NI & 128 & 10 & 138 & 169 & 307 & 0.35 & 873 & $(775-970)$ \\
\hline \multicolumn{9}{|l|}{ Scotland } \\
\hline Abrdn & 231 & 21 & 252 & 305 & 557 & 0.60 & 928 & $(851-1,006)$ \\
\hline Airdrie & 185 & 24 & 209 & 231 & 440 & 0.55 & 797 & $(723-872)$ \\
\hline D \& Gall & 50 & 10 & 60 & 71 & 131 & 0.15 & 883 & $(731-1,034)$ \\
\hline Dundee & 179 & 21 & 200 & 220 & 420 & 0.46 & 907 & $(820-993)$ \\
\hline Edinb $^{a}$ & 289 & 37 & 326 & 454 & 780 & 0.96 & 809 & (752-866) \\
\hline Glasgw $^{\mathrm{a}}$ & 593 & 54 & 647 & 1,107 & 1,754 & 1.62 & 1,080 & $(1,030-1,131)$ \\
\hline Inverns & 93 & 11 & 104 & 156 & 260 & 0.27 & 963 & $(846-1,080)$ \\
\hline Klmarnk & 141 & 33 & 174 & 144 & 318 & 0.36 & 880 & $(783-977)$ \\
\hline Krkcldy & 144 & 18 & 162 & 133 & 295 & 0.32 & 931 & $(825-1,038)$ \\
\hline \multicolumn{9}{|l|}{ Wales } \\
\hline Bangor & 75 & 16 & 91 & 0 & 91 & 0.22 & 417 & (331-503) \\
\hline Cardff $^{\mathrm{a}}$ & 517 & 75 & 592 & 1,038 & 1,630 & 1.42 & 1,148 & $(1,092-1,204)$ \\
\hline Clwyd & 73 & 15 & 88 & 90 & 178 & 0.19 & 939 & $(801-1,076)$ \\
\hline Swanse & 373 & 67 & 440 & 328 & 768 & 0.89 & 867 & (806-929) \\
\hline Wrexm & 124 & 33 & 157 & 153 & 310 & 0.24 & 1,290 & $(1,147-1,434)$ \\
\hline England & 21,560 & 3,103 & 24,663 & 28,698 & 53,361 & & & \\
\hline N Ireland & 634 & 77 & 711 & 1,069 & 1,780 & & & \\
\hline Scotland & 1,905 & 229 & 2,134 & 2,821 & 4,955 & & & \\
\hline Wales & 1,162 & 206 & 1,368 & 1,698 & 3,066 & & & \\
\hline UK & 25,261 & 3,615 & 28,876 & 34,286 & 63,162 & & & \\
\hline
\end{tabular}

Blank cells indicate no patients on that treatment type attending that centre when data were collected

Centres prefixed 'L' are London centres

The numbers of patients calculated for each country quoted above differ marginally from those quoted elsewhere in this report when patients are allocated to areas by their individual post codes, because some centres treat patients from across national boundaries

${ }^{\mathrm{a}}$ Transplant centre

${ }^{\mathrm{b}}$ Cambridge was unable to submit 2016 data at patient level but provided summary numbers of patients still on RRT at the end of 2016, by treatment modality and prevalent numbers. This centre is therefore excluded from all centre level prevalent analyses. Tables $2.1,2.2,2.3$ and 2.4 reflect these revisions: $\mathrm{Camb}(+1,551)$

figures D3 and D4 in appendix D (www.renalreg.org) can be used to determine if a CCG/HB falls within the range representing the $95 \%$ confidence limit of the national average prevalence.

Factors associated with variation in SPRs in CCGs in England, Health and Social Care Trust Areas in

Northern Ireland (HBs), Local Health Boards in

Wales (HBs) and Health Boards in Scotland (HBs)

In 2016, there were 77 CCGs/HBs with a significantly low SPR, 103 with a 'normal' SPR and 45 with a significantly high SPR (table 2.5). Prevalence ratios were not estimated for eight CCGs where more than 15\% of patients were estimated to be treated at the Cambridge renal centre which was unable to provide patient-level data.

As seen in previous years, SPRs tended to reflect the demographics of the regions in question such that urban, ethnically diverse populations in areas of high social deprivation had the highest prevalence of RRT. For example, the association with the level of ethnic diversity is illustrated by the fact that mean SPRs were significantly higher in the $84 \mathrm{CCGs} / \mathrm{HBs}$ with an ethnic minority population greater than $10 \%$ than in those with lower ethnic minority populations $(p<0.001)$. There was a strong, positive correlation between the 
Table 2.3. Number of prevalent patients on RRT by centre at year end 2012-2016

\begin{tabular}{|c|c|c|c|c|c|c|c|}
\hline \multirow[b]{2}{*}{ Centre } & \multicolumn{5}{|c|}{ Date } & \multirow{2}{*}{$\begin{array}{c}\text { \% change } \\
2015-2016\end{array}$} & \multirow{2}{*}{$\begin{array}{r}\% \text { annu } \\
\text { change } \\
2012-20\end{array}$} \\
\hline & $31 / 12 / 2012$ & $31 / 12 / 2013$ & $31 / 12 / 2014$ & $31 / 12 / 2015$ & $31 / 12 / 2016$ & & \\
\hline \multicolumn{8}{|l|}{ England } \\
\hline B Heart & 668 & 654 & 635 & 654 & 654 & 0.0 & -0.5 \\
\hline B QEH & 1,970 & 2,045 & 2,134 & 2,247 & 2,394 & 6.5 & 5.0 \\
\hline Basldn & 258 & 270 & 278 & 274 & 276 & 0.7 & 1.7 \\
\hline Bradfd & 504 & 520 & 548 & 584 & 635 & 8.7 & 5.9 \\
\hline Brightn & 829 & 870 & 914 & 950 & 996 & 4.8 & 4.7 \\
\hline Bristol & 1,338 & 1,424 & 1,458 & 1,477 & 1,470 & -0.5 & 2.4 \\
\hline Camb & 1,111 & 1,191 & 1,241 & 1,539 & 1,551 & 0.8 & 8.7 \\
\hline Carlis & 216 & 227 & 250 & 281 & 279 & -0.7 & 6.6 \\
\hline Carsh & 1,455 & 1,479 & 1,551 & 1,582 & 1,641 & 3.7 & 3.1 \\
\hline Chelms & 225 & 241 & 261 & 288 & 278 & -3.5 & 5.4 \\
\hline Colchr & 117 & 115 & 119 & 120 & 124 & 3.3 & 1.5 \\
\hline Covnt & 899 & 929 & 960 & 961 & 977 & 1.7 & 2.1 \\
\hline Derby & 474 & 464 & 513 & 538 & 543 & 0.9 & 3.5 \\
\hline Donc & 261 & 259 & 284 & 302 & 330 & 9.3 & 6.0 \\
\hline Dorset & 609 & 627 & 664 & 681 & 687 & 0.9 & 3.1 \\
\hline Dudley & 315 & 310 & 305 & 314 & 346 & 10.2 & 2.4 \\
\hline Exeter & 842 & 888 & 945 & 968 & 1,017 & 5.1 & 4.8 \\
\hline Glouc & 415 & 410 & 428 & 443 & 470 & 6.1 & 3.2 \\
\hline Hull & 782 & 813 & 801 & 857 & 858 & 0.1 & 2.3 \\
\hline Ipswi & 339 & 355 & 367 & 403 & 411 & 2.0 & 4.9 \\
\hline Kent & 918 & 958 & 1,013 & 1,039 & 1,070 & 3.0 & 3.9 \\
\hline L Barts & 1,947 & 2,090 & 2,208 & 2,278 & 2,372 & 4.1 & 5.1 \\
\hline L Guys & 1,738 & 1,828 & 1,913 & 2,012 & 2,098 & 4.3 & 4.8 \\
\hline L Kings & 917 & 963 & 1,023 & 1,084 & 1,108 & 2.2 & 4.8 \\
\hline L Rfree & 1,841 & 1,921 & 2,006 & 2,093 & 2,177 & 4.0 & 4.3 \\
\hline L St.G & 705 & 755 & 793 & 846 & 863 & 2.0 & 5.2 \\
\hline L West & 3,078 & 3,121 & 3,231 & 3,315 & 3,417 & 3.1 & 2.6 \\
\hline Leeds & 1,413 & 1,464 & 1,500 & 1,523 & 1,552 & 1.9 & 2.4 \\
\hline Leic & 1,974 & 2,067 & 2,145 & 2,184 & 2,310 & 5.8 & 4.0 \\
\hline Liv Ain & 194 & 190 & 217 & 221 & 227 & 2.7 & 4.0 \\
\hline Liv Roy & 1,228 & 1,263 & 1,268 & 1,237 & 1,225 & -1.0 & -0.1 \\
\hline M RI & 1,710 & 1,854 & 1,795 & 1,890 & 1,994 & 5.5 & 3.9 \\
\hline Middlbr & 788 & 827 & 854 & 902 & 891 & -1.2 & 3.1 \\
\hline Newc & 946 & 962 & 977 & 1,009 & 1,053 & 4.4 & 2.7 \\
\hline Norwch & 622 & 690 & 687 & 740 & 774 & 4.6 & 5.6 \\
\hline Nottm & 1,012 & 1,073 & 1,061 & 1,113 & 1,152 & 3.5 & 3.3 \\
\hline Oxford & 1,532 & 1,563 & 1,655 & 1,691 & 1,767 & 4.5 & 3.6 \\
\hline Plymth & 458 & 502 & 502 & 503 & 513 & 2.0 & 2.9 \\
\hline Ports & 1,439 & 1,544 & 1,591 & 1,669 & 1,693 & 1.4 & 4.1 \\
\hline Prestn & 1,079 & 1,089 & 1,171 & 1,215 & 1,206 & -0.7 & 2.8 \\
\hline Redng & 672 & 731 & 760 & 775 & 794 & 2.5 & 4.3 \\
\hline Salford & 880 & 881 & 971 & 974 & 1,022 & 4.9 & 3.8 \\
\hline Sheff & 1,299 & 1,328 & 1,361 & 1,384 & 1,427 & 3.1 & 2.4 \\
\hline Shrew & 354 & 338 & 349 & 368 & 375 & 1.9 & 1.5 \\
\hline Stevng & 664 & 755 & 778 & 817 & 904 & 10.6 & 8.0 \\
\hline Sthend & 213 & 220 & 238 & 246 & 237 & -3.7 & 2.7 \\
\hline Stoke & 699 & 724 & 775 & 788 & 827 & 4.9 & 4.3 \\
\hline Sund & 422 & 421 & 450 & 459 & 507 & 10.5 & 4.7 \\
\hline Truro & 375 & 371 & 379 & 415 & 428 & 3.1 & 3.4 \\
\hline Wirral & 226 & 248 & 277 & 280 & 337 & 20.4 & 10.5 \\
\hline Wolve & 524 & 567 & 574 & 582 & 569 & -2.2 & 2.1 \\
\hline York & 396 & 409 & 461 & 490 & 535 & 9.2 & 7.8 \\
\hline
\end{tabular}


Table 2.3. Continued

\begin{tabular}{|c|c|c|c|c|c|c|c|}
\hline \multirow[b]{2}{*}{ Centre } & \multicolumn{5}{|c|}{ Date } & \multirow{2}{*}{$\begin{array}{c}\text { \% change } \\
2015-2016\end{array}$} & \multirow{2}{*}{$\begin{array}{l}\% \text { annual } \\
\text { change } \\
2012-2016\end{array}$} \\
\hline & $31 / 12 / 2012$ & $31 / 12 / 2013$ & $31 / 12 / 2014$ & $31 / 12 / 2015$ & $31 / 12 / 2016$ & & \\
\hline Antrim & 223 & 224 & 229 & 239 & 241 & 0.8 & 2.0 \\
\hline Belfast & 702 & 726 & 747 & 772 & 829 & 7.4 & 4.2 \\
\hline Newry & 188 & 199 & 208 & 226 & 237 & 4.9 & 6.0 \\
\hline \multicolumn{8}{|l|}{ Scotland } \\
\hline Abrdn & 507 & 517 & 502 & 532 & 557 & 4.7 & 2.4 \\
\hline Airdrie & 389 & 389 & 395 & 425 & 440 & 3.5 & 3.1 \\
\hline D \& Gall & 128 & 119 & 130 & 130 & 131 & 0.8 & 0.6 \\
\hline Dundee & 395 & 398 & 401 & 420 & 420 & 0.0 & 1.5 \\
\hline Krkcldy & 278 & 283 & 277 & 295 & 295 & 0.0 & 1.5 \\
\hline \multicolumn{8}{|l|}{ Wales } \\
\hline Bangor & 105 & 99 & 102 & 182 & 177 & -2.7 & 13.9 \\
\hline Cardff & 1,544 & 1,583 & 1,591 & 1,612 & 1,630 & 1.1 & 1.4 \\
\hline Clwyd & 173 & 152 & 166 & 185 & 178 & -3.8 & 0.7 \\
\hline Swanse & 663 & 692 & 707 & 766 & 768 & 0.3 & 3.7 \\
\hline Wrexm & 248 & 251 & 282 & 293 & 310 & 5.8 & 5.7 \\
\hline England & 45,890 & 47,808 & 49,639 & 51,605 & 53,361 & 3.4 & 3.8 \\
\hline N Ireland & 1,512 & 1,542 & 1,607 & 1,701 & 1,780 & 4.6 & 4.2 \\
\hline Scotland & 4,474 & 4,541 & 4,583 & 4,845 & 4,955 & 2.3 & 2.6 \\
\hline Wales & 2,733 & 2,777 & 2,848 & 3,038 & 3,066 & 0.9 & 2.9 \\
\hline
\end{tabular}

SPR and percentage of the population that were nonWhite $(r=0.9, p<0.001)$. In 2016 , for each $10 \%$ increase in ethnic minority population, the SPR increased by 0.17 (equates to $\sim 17 \%$ ). These trends are identical to those identified previously. The relationship between the

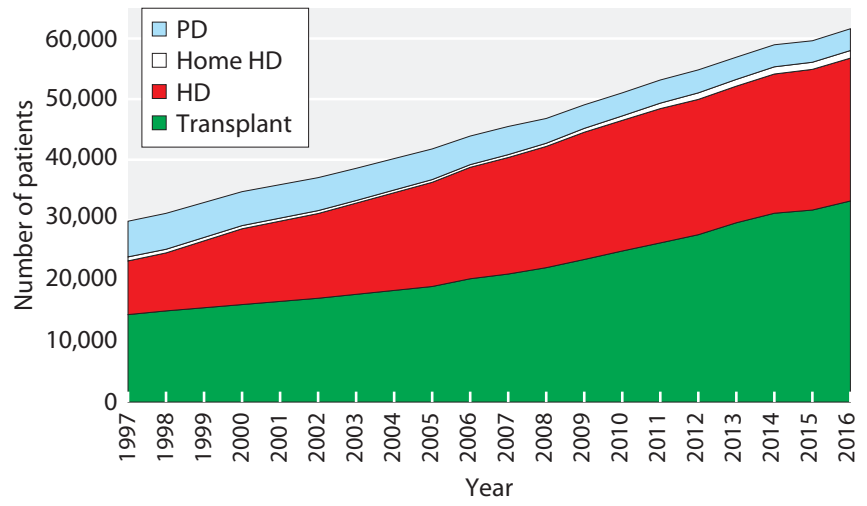

Fig. 2.2. Growth in prevalent patients by treatment modality at the end of each year 1997-2016

UK RRT prevalence in 2016 ethnic composition of a CCG/HB and its SPR is demonstrated in figure 2.3.

Excluding the eight CCGs where $\geqslant 15 \%$ of their population was covered by Cambridge, only three of the 139 CCGs/HBs with ethnic minority populations of less than $10 \%$ had high SPRs: Belfast in Northern Ireland, Cwm Taf and Abertawe Bro Morgannwg University in Wales. Of the 86 CCGs/HBs with ethnic minority populations greater than or equal to $10 \%, 42(48.8 \%)$ had high SPRs, whereas eleven (12.8\%) (NHS Chiltern, NHS Leeds North, NHS Leeds West, NHS Richmond, NHS Havering, NHS Solihull, NHS Calderdale, NHS Newcastle and Gateshead, NHS East and North Hertfordshire, NHS Medway, NHS Trafford) had low SPRs. Some of the CCGs/HBs with a high $(>15 \%)$ ethnic minority population had a normal expected RRT prevalence (e.g. NHS Central London (Westminster), NHS Wolverhampton, NHS Sheffield, NHS Crawley). Therefore, although differences in proportions of ethnic minority populations

Nephron 2018;139(suppl1):47-74 
Table 2.4. Change in RRT prevalence ratio pmp 2012-2016 by modality*

\begin{tabular}{|c|c|c|c|c|c|c|c|c|c|c|}
\hline \multirow[b]{2}{*}{ Year } & \multicolumn{5}{|c|}{ Prevalence } & \multicolumn{5}{|c|}{$\%$ growth in prevalence pmp } \\
\hline & HD pmp & $\mathrm{PD}$ pmp & Dialysis pmp & Transplant pmp & RRT pmp & HD & $\mathrm{PD}$ & Dialysis & Transplant & RRT \\
\hline 2013 & 369 & 57 & 427 & 462 & 888 & -0.1 & -4.6 & -0.8 & 5.8 & 2.5 \\
\hline 2014 & 374 & 56 & 430 & 482 & 913 & 1.3 & -1.5 & 0.9 & 4.5 & 2.8 \\
\hline 2015 & 384 & 55 & 440 & 501 & 941 & 2.7 & -1.6 & 2.2 & 3.9 & 3.1 \\
\hline
\end{tabular}

${ }^{*}$ Differences in the figures for dialysis and RRT prevalence and the sum of the separate modalities are due to rounding pmp - per million population

are clearly important in explaining differences in SPR they are not the only explanation.

The age and sex SPRs (which do not take into account variation in ethnicity) in each region of England and in Wales, Northern Ireland and Scotland are presented in table 2.6. Wales and Northern Ireland previously had higher than expected RRT prevalence but in more recent years were similar to expected. Scotland had lower than expected RRT prevalence as did the North and South of England. RRT prevalence in London remained higher than expected.

\section{Case mix in prevalent $R R T$ patients}

Time on RRT (vintage)

Table 2.7 shows the median time, in years, since starting RRT of prevalent RRT patients on 31 December 2016. Median time on RRT for all prevalent patients has remained fairly static at 6.2 years (6.2 years in 2015, 6.1 years in 2014). Patients with functioning transplants had survived a median of 10.3 years on RRT whilst the median time on RRT of HD and PD patients was significantly less (3.2 and 1.5 years respectively). The median time on HD was more than double that on PD and this could reflect early transplantation in the latter as well as higher technique failure rates for PD.

$$
\text { Age }
$$

The median age of prevalent UK patients on RRT at 31 December 2016 (59.1 years, table 2.8) has remained stable over recent years although it is significantly higher than in 2005 when it was 55.0 years. As observed previously, there were marked differences between modalities; the median age of HD patients (67.2 years) was greater than that of those on PD (63.7 years) and substantially higher than that of transplanted patients (54.3 years). Of the UK prevalent RRT population, $49.9 \%$ were in the 40-64 years age group (table 2.9). The proportion of patients aged 75 years and older varied greatly between countries and was highest in Wales (17.8\%) and Northern Ireland (18.4\%) and lowest in Scotland (12.5\%) (table 2.9). Within countries there were large differences in the proportion of patients aged over 75 (within England these ranged between $8.8 \%$ in Liverpool Royal Infirmary and $41.4 \%$ in Colchester). In most centres the prevalent PD population was younger than the HD population (table 2.8).

Inter-centre differences in the median age of prevalent patients by treatment modality can reflect differing demographics of the catchment populations as well as differing approaches to treatment modalities. For example, Colchester had the highest median age (72.0 years), whilst London Guy's the lowest (55.3 years) (table 2.8). This could reflect either variation in the catchment populations or follow-up of younger transplant patients (Colchester had no transplant patients whereas $65 \%$ of prevalent patients at London Guy's were transplant patients). The median age of the non-White dialysis population was lower than the overall dialysis population (62.0 vs 66.8 years, data not shown). The differing age distributions of the transplant and dialysis populations are illustrated in figure 2.4, demonstrating that the age peak for prevalent dialysis patients was 24 years later than for prevalent transplant patients.

In the UK on 31 December 2016, 66.3\% of patients on RRT aged less than 65 years had a functioning transplant (table 2.15), compared with only $32.6 \%$ of those aged 65 years and over. There was a similar pattern in all four UK countries, although the proportion of patients aged less than 65 years with a functioning transplant in Northern Ireland (77.4\%) was much higher than elsewhere.

Sex

The age distributions of males and females were very similar (data not shown). Standardising the age of the 
Table 2.5. Prevalence of RRT and SPRs in CCG/HB area

$\mathrm{O} / \mathrm{E}$ - ratio of observed:expected rate of RRT given the age and sex breakdown of the area

LCL - lower 95\% confidence limit

UCL - upper 95\% confidence limit

Areas with significantly low SPRs in 2016 are italicised in lighter greyed areas, those with significantly high SPRs in 2016 are bold in darker greyed areas

Population numbers are the 2016 mid-year estimates by age group and sex (data obtained from the Office of National Statistics, National Records of Scotland and the Northern Ireland Statistics and Research Agency - based on the 2011 census)

$\%$ non-White - percentage of the CCG/HB population that is non-White, from 2011 census

Office of National Statistics specifies that the populations should be rounded to the nearest 100 when presented

\begin{tabular}{|c|c|c|c|c|c|c|c|c|c|c|c|c|}
\hline UK area & Name & $\begin{array}{c}\text { Total } \\
\text { population }\end{array}$ & $\begin{array}{c}2011 \\
\mathrm{O} / \mathrm{E}\end{array}$ & $\begin{array}{c}2012 \\
\mathrm{O} / \mathrm{E}\end{array}$ & $\begin{array}{l}2013 \\
\mathrm{O} / \mathrm{E}\end{array}$ & $\begin{array}{l}2014 \\
\mathrm{O} / \mathrm{E}\end{array}$ & $\begin{array}{l}2015 \\
\mathrm{O} / \mathrm{E}\end{array}$ & $\begin{array}{l}2016 \\
\mathrm{O} / \mathrm{E}\end{array}$ & $\begin{array}{l}95 \% \\
\text { LCL }\end{array}$ & $\begin{array}{r}20 \\
95 \% \\
\text { UCL } \\
\end{array}$ & $\begin{array}{c}16 \\
\text { Crude rate } \\
\text { pmp }\end{array}$ & $\begin{array}{c}\% \\
\text { non- } \\
\text { White }\end{array}$ \\
\hline \multirow{4}{*}{$\begin{array}{l}\text { Cheshire, } \\
\text { Warrington } \\
\text { and Wirral }\end{array}$} & NHS Eastern Cheshire & 196,900 & 0.76 & 0.81 & 0.78 & 0.78 & 0.77 & 0.72 & 0.62 & 0.84 & 792 & 3.7 \\
\hline & NHS Vale Royal & 103,700 & 0.78 & 0.73 & 0.79 & 0.74 & 0.74 & 0.69 & 0.55 & 0.86 & 704 & 2.1 \\
\hline & NHS Warrington & 208,800 & 0.80 & 0.82 & 0.84 & 0.91 & 0.87 & 0.83 & 0.71 & 0.96 & 819 & 4.1 \\
\hline & NHS West Cheshire & 232,000 & 0.99 & 0.96 & 0.97 & 0.95 & 0.83 & 0.86 & 0.75 & 0.98 & 892 & 2.8 \\
\hline \multirow{5}{*}{$\begin{array}{l}\text { Durham, } \\
\text { Darlington } \\
\text { and Tees }\end{array}$} & NHS Darlington & 105,600 & 0.80 & 0.85 & 0.85 & 0.84 & 0.87 & 0.85 & 0.69 & 1.04 & 852 & 3.8 \\
\hline & $\begin{array}{l}\text { NHS Durham Dales, Easington and } \\
\text { Sedgefield }\end{array}$ & 274,600 & 0.99 & 0.95 & 0.98 & 0.98 & 0.98 & 0.95 & 0.85 & 1.07 & 998 & 1.2 \\
\hline & NHS Hartlepool and Stockton-on-Tees & 288,500 & 0.89 & 0.91 & 0.89 & 0.92 & 0.88 & 0.84 & 0.74 & 0.96 & 818 & 4.4 \\
\hline & NHS North Durham & 247,500 & 0.76 & 0.84 & 0.80 & 0.79 & 0.78 & 0.77 & 0.67 & 0.89 & 776 & 2.5 \\
\hline & NHS South Tees & 275,800 & 1.12 & 1.11 & 1.11 & 1.07 & 1.13 & 1.08 & 0.97 & 1.22 & 1,051 & 6.7 \\
\hline \multirow{7}{*}{$\begin{array}{l}\text { Greater } \\
\text { Manchester }\end{array}$} & NHS Manchester & 541,300 & 1.11 & 1.16 & 1.19 & 1.22 & 1.24 & 1.26 & 1.15 & 1.37 & 896 & 33.5 \\
\hline & NHS Oldham & 232,700 & 0.94 & 0.93 & 0.96 & 0.95 & 0.99 & 1.02 & 0.90 & 1.17 & 915 & 22.5 \\
\hline & NHS Salford & 248,700 & 0.85 & 0.88 & 0.90 & 0.87 & 0.83 & 0.89 & 0.77 & 1.02 & 772 & 9.9 \\
\hline & NHS Stockport & 290,600 & 0.88 & 0.87 & 0.80 & 0.81 & 0.82 & 0.83 & 0.73 & 0.94 & 833 & 7.9 \\
\hline & NHS Tameside and Glossop & 256,400 & 0.94 & 0.94 & 0.93 & 0.91 & 0.89 & 0.94 & 0.83 & 1.07 & 913 & 8.2 \\
\hline & NHS Trafford & 234,700 & 0.84 & 0.85 & 0.87 & 0.88 & 0.84 & 0.83 & 0.72 & 0.96 & 793 & 14.5 \\
\hline & NHS Wigan Borough & 323,100 & 0.90 & 0.94 & 0.96 & 0.97 & 0.92 & 0.93 & 0.83 & 1.04 & 932 & 2.7 \\
\hline \multirow[t]{4}{*}{ Lancashire } & NHS Blackburn with Darwen & 147,000 & 1.30 & 1.27 & 1.26 & 1.22 & 1.24 & 1.20 & 1.03 & 1.41 & 1,047 & 30.8 \\
\hline & NHS Blackpool & 139,200 & 0.78 & 0.88 & 0.96 & 1.04 & 1.01 & 0.98 & 0.83 & 1.15 & 999 & 3.3 \\
\hline & NHS Chorley and South Ribble & 174,300 & 0.81 & 0.87 & 0.94 & 0.92 & 0.89 & 0.87 & 0.74 & 1.02 & 878 & 2.9 \\
\hline & NHS East Lancashire & 375,800 & 1.03 & 0.99 & 1.01 & 1.01 & 0.98 & 0.97 & 0.88 & 1.08 & 955 & 11.9 \\
\hline \multirow{3}{*}{ Merseyside } & NHS South Sefton & 158,900 & 0.94 & 0.95 & 0.95 & 0.98 & 0.97 & 0.99 & 0.84 & 1.15 & 1,013 & 2.2 \\
\hline & NHS Southport and Formby & 115,400 & 0.83 & 0.76 & 0.80 & 0.81 & 0.76 & 0.75 & 0.62 & 0.92 & 841 & 3.1 \\
\hline & NHS St Helens & 178,500 & 0.90 & 0.92 & 0.86 & 0.86 & 0.85 & 0.85 & 0.72 & 0.99 & 863 & 2.0 \\
\hline \multirow{6}{*}{$\begin{array}{l}\text { Cumbria, } \\
\text { Northum- } \\
\text { berland, } \\
\text { Tyne and } \\
\text { Wear }\end{array}$} & NHS Cumbria North & 318,200 & 0.73 & 0.70 & 0.73 & 0.76 & 0.81 & 0.80 & 0.71 & 0.90 & 870 & 1.5 \\
\hline & NHS Newcastle Gateshead & 498,100 & 0.89 & 0.88 & 0.84 & 0.84 & 0.84 & 0.85 & 0.77 & 0.94 & 769 & 10.1 \\
\hline & NHS North Tyneside & 203,300 & 0.93 & 0.94 & 0.97 & 0.90 & 0.88 & 0.91 & 0.79 & 1.05 & 930 & 3.4 \\
\hline & NHS Northumberland & 316,000 & 0.74 & 0.74 & 0.72 & 0.77 & 0.76 & 0.73 & 0.65 & 0.83 & 816 & 1.6 \\
\hline & NHS South Tyneside & 149,400 & 1.04 & 0.98 & 0.94 & 0.86 & 0.84 & 0.95 & 0.81 & 1.12 & 970 & 4.1 \\
\hline & NHS Sunderland & 278,000 & 0.98 & 1.00 & 0.95 & 0.96 & 0.94 & 1.01 & 0.90 & 1.14 & 1,011 & 4.1 \\
\hline
\end{tabular}


Table 2.5. Continued

\begin{tabular}{|c|c|c|c|c|c|c|c|c|c|c|c|c|}
\hline UK area & Name & $\begin{array}{c}\text { Total } \\
\text { population }\end{array}$ & $\begin{array}{c}2011 \\
\mathrm{O} / \mathrm{E}\end{array}$ & $\begin{array}{c}2012 \\
\mathrm{O} / \mathrm{E}\end{array}$ & $\begin{array}{c}2013 \\
\mathrm{O} / \mathrm{E}\end{array}$ & $\begin{array}{c}2014 \\
\mathrm{O} / \mathrm{E}\end{array}$ & $\begin{array}{c}2015 \\
\mathrm{O} / \mathrm{E}\end{array}$ & $\begin{array}{c}2016 \\
O / E\end{array}$ & $\begin{array}{l}95 \% \\
\text { LCL }\end{array}$ & $\begin{array}{r}201 \\
95 \% \\
\text { UCL }\end{array}$ & $\begin{array}{l}\text { Crude rate } \\
\text { pmp }\end{array}$ & $\begin{array}{c}\% \\
\text { non- } \\
\text { White }\end{array}$ \\
\hline \multirow{5}{*}{$\begin{array}{l}\text { North } \\
\text { Yorkshire } \\
\text { and Humber }\end{array}$} & NHS East Riding of Yorkshire & 315,900 & 0.85 & 0.83 & 0.81 & 0.80 & 0.80 & 0.79 & 0.71 & 0.89 & 896 & 1.9 \\
\hline & NHS Harrogate and Rural District & 156,300 & 0.82 & 0.86 & 0.84 & 0.88 & 0.93 & 0.91 & 0.78 & 1.06 & 985 & 3.7 \\
\hline & NHS Hull & 260,200 & 1.00 & 0.95 & 0.97 & 1.02 & 1.08 & 1.10 & 0.97 & 1.24 & 972 & 5.9 \\
\hline & NHS Scarborough and Ryedale & 111,400 & 0.90 & 0.94 & 0.91 & 0.89 & 0.87 & 0.88 & 0.73 & 1.06 & 979 & 2.5 \\
\hline & NHS Vale of York & 357,900 & 0.88 & 0.93 & 0.92 & 0.90 & 0.88 & 0.90 & 0.81 & 1.01 & 900 & 4.0 \\
\hline \multirow{3}{*}{$\begin{array}{l}\text { South } \\
\text { Yorkshire } \\
\text { and } \\
\text { Bassetlaw }\end{array}$} & NHS Barnsley & 241,200 & 1.11 & 1.05 & 1.02 & 1.03 & 0.99 & 1.02 & 0.90 & 1.16 & 1,028 & 2.1 \\
\hline & NHS Bassetlaw & 114,800 & 0.83 & 0.89 & 0.83 & 0.83 & 0.84 & 0.83 & 0.68 & 1.00 & 879 & 2.6 \\
\hline & NHS Doncaster & 306,400 & 0.98 & 0.96 & 0.93 & 0.96 & 0.95 & 0.97 & 0.87 & 1.09 & 960 & 4.7 \\
\hline \multirow{9}{*}{$\begin{array}{l}\text { West } \\
\text { Yorkshire }\end{array}$} & NHS Bradford City & 84,900 & 1.80 & 1.88 & 1.94 & 2.15 & 2.14 & 2.31 & 1.93 & 2.75 & 1,449 & 72.2 \\
\hline & NHS Bradford Districts & 339,700 & 1.17 & 1.24 & 1.22 & 1.19 & 1.22 & 1.28 & 1.16 & 1.41 & 1,110 & 28.7 \\
\hline & NHS Calderdale & 209,800 & 1.02 & 0.95 & 0.90 & 0.85 & 0.86 & 0.85 & 0.73 & 0.98 & 834 & 10.3 \\
\hline & NHS Greater Huddersfield & 245,000 & 0.92 & 0.98 & 0.95 & 0.97 & 0.96 & 0.94 & 0.82 & 1.07 & 898 & 17.4 \\
\hline & NHS Leeds North & 201,200 & 1.00 & 0.95 & 0.90 & 0.87 & 0.88 & 0.86 & 0.74 & 1.00 & 840 & 17.4 \\
\hline & NHS Leeds South and East & 253,700 & 0.96 & 0.95 & 0.95 & 0.98 & 0.97 & 0.97 & 0.85 & 1.11 & 812 & 18.3 \\
\hline & NHS Leeds West & 326,900 & 0.82 & 0.80 & 0.86 & 0.89 & 0.90 & 0.88 & 0.78 & 1.00 & 731 & 10.8 \\
\hline & NHS North Kirklees & 192,000 & 1.17 & 1.14 & 1.24 & 1.23 & 1.17 & 1.20 & 1.04 & 1.37 & 1,083 & 25.3 \\
\hline & NHS Wakefield & 336,800 & 0.86 & 0.88 & 0.87 & 0.86 & 0.82 & 0.83 & 0.74 & 0.93 & 828 & 4.6 \\
\hline $\begin{array}{l}\text { Arden, } \\
\text { Hereford- } \\
\text { shire and } \\
\text { Worcester- } \\
\text { shire }\end{array}$ & NHS Coventry and Rugby & 456,700 & 1.24 & 1.29 & 1.27 & 1.22 & 1.15 & 1.16 & 1.06 & 1.27 & 994 & 22.2 \\
\hline \multirow{6}{*}{$\begin{array}{l}\text { Birmingham } \\
\text { and the } \\
\text { Black } \\
\text { Country }\end{array}$} & NHS Birmingham South and Central & 204,000 & 1.69 & 1.74 & 1.73 & 1.71 & 1.66 & 1.69 & 1.50 & 1.90 & 1,324 & 40.4 \\
\hline & NHS Dudley & 317,600 & 0.90 & 0.96 & 0.97 & 0.95 & 0.94 & 0.95 & 0.85 & 1.06 & 948 & 10.0 \\
\hline & NHS Sandwell and West Birmingham & 495,100 & 1.76 & 1.72 & 1.70 & 1.67 & 1.69 & 1.77 & 1.65 & 1.91 & 1,450 & 45.3 \\
\hline & NHS Solihull & 211,800 & 0.92 & 0.89 & 0.86 & 0.83 & 0.85 & 0.84 & 0.72 & 0.97 & 850 & 10.9 \\
\hline & NHS Walsall & 278,700 & 1.34 & 1.31 & 1.32 & 1.33 & 1.30 & 1.25 & 1.12 & 1.39 & 1,162 & 21.1 \\
\hline & NHS Wolverhampton & 256,600 & 1.13 & 1.14 & 1.15 & 1.16 & 1.13 & 1.12 & 1.00 & 1.27 & 1,025 & 32.0 \\
\hline \multirow{10}{*}{$\begin{array}{l}\text { Derbyshire } \\
\text { and } \\
\text { Nottingham- } \\
\text { shire }\end{array}$} & NHS Erewash & 96,700 & 1.01 & 0.99 & 0.92 & 0.89 & 0.97 & 0.95 & 0.78 & 1.17 & 951 & 3.2 \\
\hline & NHS Hardwick & 111,400 & 0.77 & 0.74 & 0.72 & 0.73 & 0.74 & 0.74 & 0.60 & 0.91 & 772 & 1.8 \\
\hline & NHS Mansfield \& Ashfield & 197,900 & 0.96 & 0.92 & 0.93 & 0.94 & 0.93 & 0.92 & 0.80 & 1.07 & 925 & 2.5 \\
\hline & NHS Newark \& Sherwood & 119,700 & 1.12 & 1.07 & 1.02 & 0.98 & 0.90 & 0.84 & 0.70 & 1.02 & 894 & 2.4 \\
\hline & NHS North Derbyshire & 273,200 & 0.83 & 0.81 & 0.80 & 0.78 & 0.77 & 0.78 & 0.68 & 0.88 & 849 & 2.5 \\
\hline & NHS Nottingham City & 325,300 & 1.16 & 1.13 & 1.14 & 1.13 & 1.19 & 1.21 & 1.08 & 1.36 & 919 & 28.5 \\
\hline & NHS Nottingham North \& East & 150,300 & 0.90 & 0.91 & 0.88 & 0.85 & 0.82 & 0.84 & 0.70 & 1.00 & 851 & 6.2 \\
\hline & NHS Nottingham West & 112,700 & 0.99 & 1.04 & 1.07 & 1.07 & 1.06 & 1.04 & 0.87 & 1.24 & 1,065 & 7.3 \\
\hline & NHS Rushcliffe & 115,200 & 0.87 & 0.77 & 0.83 & 0.78 & 0.74 & 0.73 & 0.59 & 0.90 & 755 & 6.9 \\
\hline & NHS Southern Derbyshire & 527,400 & 1.02 & 0.98 & 0.98 & 0.99 & 0.99 & 0.99 & 0.91 & 1.08 & 961 & 11.0 \\
\hline
\end{tabular}


Table 2.5. Continued

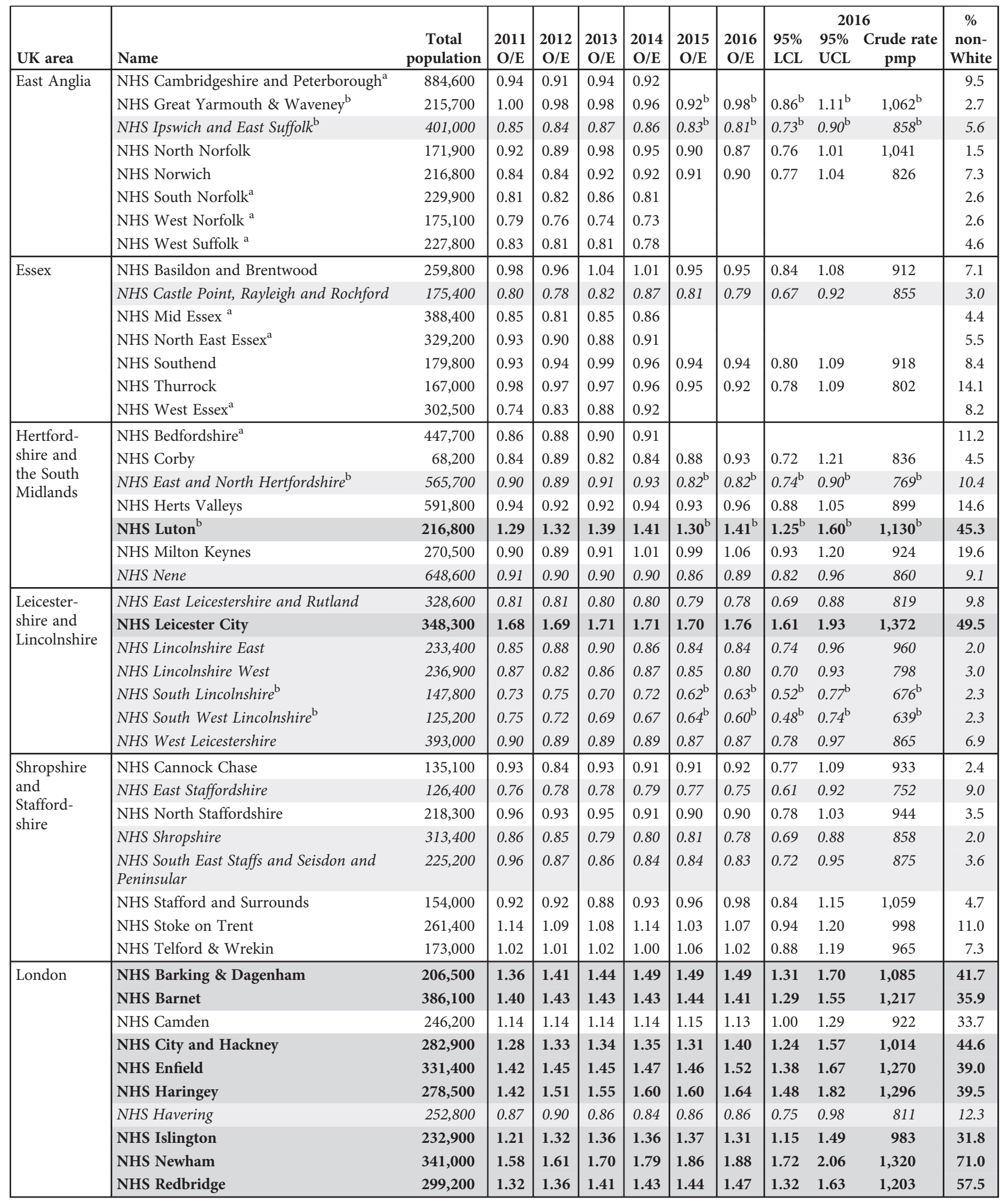


Table 2.5. Continued

\begin{tabular}{|c|c|c|c|c|c|c|c|c|c|c|c|c|}
\hline \multirow[b]{2}{*}{ UK area } & \multirow[b]{2}{*}{ Name } & \multirow[b]{2}{*}{$\begin{array}{c}\text { Total } \\
\text { population }\end{array}$} & \multirow[b]{2}{*}{$\begin{array}{c}2011 \\
\mathrm{O} / \mathrm{E}\end{array}$} & \multirow[b]{2}{*}{$\begin{array}{c}2012 \\
\mathrm{O} / \mathrm{E}\end{array}$} & \multirow[b]{2}{*}{$\begin{array}{c}2013 \\
\mathrm{O} / \mathrm{E}\end{array}$} & \multirow[b]{2}{*}{$\begin{array}{c}2014 \\
\mathrm{O} / \mathrm{E}\end{array}$} & \multirow[b]{2}{*}{$\begin{array}{c}2015 \\
\mathrm{O} / \mathrm{E}\end{array}$} & \multirow[b]{2}{*}{$\begin{array}{c}2016 \\
\text { O/E }\end{array}$} & \multicolumn{3}{|c|}{2016} & \multirow{2}{*}{$\begin{array}{c}\% \\
\text { non- } \\
\text { White }\end{array}$} \\
\hline & & & & & & & & & $\begin{array}{l}95 \% \\
\text { LCL }\end{array}$ & $\begin{array}{l}95 \% \\
\text { UCL }\end{array}$ & $\begin{array}{l}\text { Crude rate } \\
\text { pmp }\end{array}$ & \\
\hline \multirow{21}{*}{$\begin{array}{l}\text { London } \\
\text { (cont.) }\end{array}$} & NHS Tower Hamlets & 304,900 & 1.19 & 1.28 & 1.35 & 1.41 & 1.48 & 1.49 & 1.33 & 1.67 & 991 & 54.8 \\
\hline & NHS Waltham Forest & 275,800 & 1.46 & 1.41 & 1.46 & 1.58 & 1.59 & 1.64 & 1.48 & 1.82 & 1,305 & 47.8 \\
\hline & NHS Brent & 328,300 & 2.02 & 2.06 & 2.05 & 2.09 & 2.12 & 2.14 & 1.98 & 2.32 & 1,773 & 63.7 \\
\hline & NHS Central London (Westminster) & 178,400 & 1.01 & 1.01 & 1.06 & 1.08 & 1.07 & 1.09 & 0.94 & 1.26 & 959 & 36.2 \\
\hline & NHS Hammersmith and Fulham & 179,700 & 1.30 & 1.31 & 1.26 & 1.30 & 1.29 & 1.37 & 1.20 & 1.58 & 1,113 & 31.9 \\
\hline & NHS Harrow & 248,800 & 1.83 & 1.82 & 1.72 & 1.71 & 1.69 & 1.73 & 1.57 & 1.91 & 1,560 & 57.8 \\
\hline & NHS Hillingdon & 302,500 & 1.44 & 1.47 & 1.48 & 1.47 & 1.42 & 1.41 & 1.27 & 1.56 & 1,187 & 39.4 \\
\hline & NHS Hounslow & 271,100 & 1.42 & 1.45 & 1.52 & 1.53 & 1.55 & 1.57 & 1.42 & 1.75 & 1,306 & 48.6 \\
\hline & $\begin{array}{l}\text { NHS West London (Kensington and } \\
\text { Chelsea, Queen's Park and Paddington) }\end{array}$ & 226,000 & 1.19 & 1.14 & 1.14 & 1.19 & 1.15 & 1.15 & 1.01 & 1.30 & 1,022 & 33.4 \\
\hline & NHS Bexley & 244,800 & 1.28 & 1.28 & 1.27 & 1.30 & 1.32 & 1.39 & 1.24 & 1.55 & 1,271 & 18.1 \\
\hline & NHS Bromley & 326,900 & 0.99 & 0.96 & 0.97 & 0.97 & 1.05 & 1.04 & 0.93 & 1.16 & 988 & 15.7 \\
\hline & NHS Croydon & 382,300 & 1.33 & 1.39 & 1.44 & 1.45 & 1.45 & 1.48 & 1.35 & 1.61 & 1,279 & 44.9 \\
\hline & NHS Greenwich & 279,800 & 1.22 & 1.21 & 1.37 & 1.39 & 1.40 & 1.45 & 1.30 & 1.62 & 1,147 & 37.5 \\
\hline & NHS Kingston & 176,100 & 1.11 & 1.09 & 1.02 & 1.03 & 0.99 & 1.05 & 0.90 & 1.23 & 903 & 25.5 \\
\hline & NHS Lambeth & 327,900 & 1.56 & 1.61 & 1.64 & 1.71 & 1.75 & 1.71 & 1.56 & 1.88 & 1,293 & 42.9 \\
\hline & NHS Lewisham & 301,900 & 1.46 & 1.49 & 1.52 & 1.50 & 1.50 & 1.49 & 1.35 & 1.66 & 1,176 & 46.5 \\
\hline & NHS Merton & 205,000 & 1.25 & 1.31 & 1.28 & 1.36 & 1.40 & 1.45 & 1.28 & 1.64 & 1,229 & 35.1 \\
\hline & NHS Richmond & 195,800 & 0.74 & 0.75 & 0.77 & 0.75 & 0.73 & 0.72 & 0.60 & 0.85 & 664 & 14.0 \\
\hline & NHS Southwark & 313,200 & 1.69 & 1.75 & 1.78 & 1.83 & 1.86 & 1.85 & 1.69 & 2.03 & 1,411 & 45.8 \\
\hline & NHS Sutton & 202,200 & 1.18 & 1.20 & 1.15 & 1.15 & 1.18 & 1.21 & 1.06 & 1.38 & 1,103 & 21.4 \\
\hline & NHS Wandsworth & 316,100 & 1.23 & 1.18 & 1.15 & 1.23 & 1.28 & 1.29 & 1.15 & 1.44 & 990 & 28.6 \\
\hline \multirow{4}{*}{$\begin{array}{l}\text { Bath, } \\
\text { Gloucester- } \\
\text { shire, } \\
\text { Swindon and } \\
\text { Wiltshire }\end{array}$} & NHS Bath and North East Somerset & 187,800 & 0.81 & 0.82 & 0.83 & 0.82 & 0.85 & 0.82 & 0.70 & 0.97 & 772 & 5.4 \\
\hline & NHS Gloucestershire & 623,100 & 0.88 & 0.89 & 0.88 & 0.87 & 0.86 & 0.88 & 0.81 & 0.95 & 907 & 4.6 \\
\hline & NHS Swindon & 223,600 & 0.91 & 0.94 & 0.95 & 0.98 & 1.00 & 0.99 & 0.86 & 1.13 & 930 & 10.0 \\
\hline & NHS Wiltshire & 488,400 & 0.75 & 0.72 & 0.73 & 0.72 & 0.73 & 0.74 & 0.67 & 0.82 & 766 & 3.4 \\
\hline \multirow{4}{*}{$\begin{array}{l}\text { Bristol, North } \\
\text { Somerset, } \\
\text { Somerset and } \\
\text { South Glou- } \\
\text { cestershire }\end{array}$} & NHS Bristol & 454,200 & 1.22 & 1.25 & 1.29 & 1.28 & 1.24 & 1.20 & 1.10 & 1.32 & 975 & 16.0 \\
\hline & NHS North Somerset & 211,700 & 0.91 & 0.95 & 0.94 & 0.95 & 0.92 & 0.87 & 0.76 & 1.00 & 931 & 2.7 \\
\hline & NHS Somerset & 549,400 & 0.87 & 0.85 & 0.82 & 0.83 & 0.80 & 0.82 & 0.75 & 0.90 & 888 & 2.0 \\
\hline & NHS South Gloucestershire & 277,600 & 0.93 & 0.91 & 0.96 & 0.95 & 0.90 & 0.87 & 0.76 & 0.99 & 850 & 5.0 \\
\hline \multirow{3}{*}{$\begin{array}{l}\text { Devon, } \\
\text { Cornwall and } \\
\text { Isles of Scilly }\end{array}$} & NHS Kernow & 556,000 & 0.99 & 0.98 & 0.98 & 0.97 & 0.96 & 0.94 & 0.86 & 1.02 & 1,023 & 1.8 \\
\hline & NHS North, East, West Devon & 898,000 & 0.93 & 0.94 & 0.93 & 0.92 & 0.91 & 0.90 & 0.84 & 0.96 & 933 & 3.0 \\
\hline & NHS South Devon and Torbay & 279,900 & 1.07 & 1.05 & 1.09 & 1.07 & 1.05 & 1.05 & 0.95 & 1.17 & 1,193 & 2.1 \\
\hline \multirow{8}{*}{$\begin{array}{l}\text { Kent and } \\
\text { Medway }\end{array}$} & NHS Ashford & 126,200 & 1.01 & 1.02 & 0.97 & 0.98 & 0.94 & 0.97 & 0.81 & 1.16 & 951 & 6.3 \\
\hline & NHS Canterbury and Coastal & 210,500 & 0.98 & 0.97 & 1.00 & 1.07 & 1.05 & 0.99 & 0.87 & 1.14 & 969 & 5.9 \\
\hline & NHS Dartford, Gravesham and Swanley & 260,600 & 1.06 & 1.08 & 1.11 & 1.13 & 1.09 & 1.09 & 0.97 & 1.23 & 1,032 & 13.0 \\
\hline & NHS Medway & 278,500 & 0.84 & 0.87 & 0.91 & 0.91 & 0.91 & 0.87 & 0.76 & 0.99 & 793 & 10.4 \\
\hline & NHS South Kent Coast & 207,600 & 0.83 & 0.83 & 0.78 & 0.82 & 0.81 & 0.83 & 0.72 & 0.96 & 896 & 4.5 \\
\hline & NHS Swale & 114,800 & 1.09 & 1.17 & 1.18 & 1.13 & 1.10 & 1.10 & 0.92 & 1.32 & 1,063 & 3.8 \\
\hline & NHS Thanet & 140,700 & 1.00 & 1.05 & 1.11 & 1.06 & 1.00 & 0.99 & 0.84 & 1.16 & 1,024 & 4.5 \\
\hline & NHS West Kent & 481,600 & 0.79 & 0.81 & 0.79 & 0.82 & 0.79 & 0.81 & 0.73 & 0.89 & 797 & 4.9 \\
\hline
\end{tabular}


Table 2.5. Continued

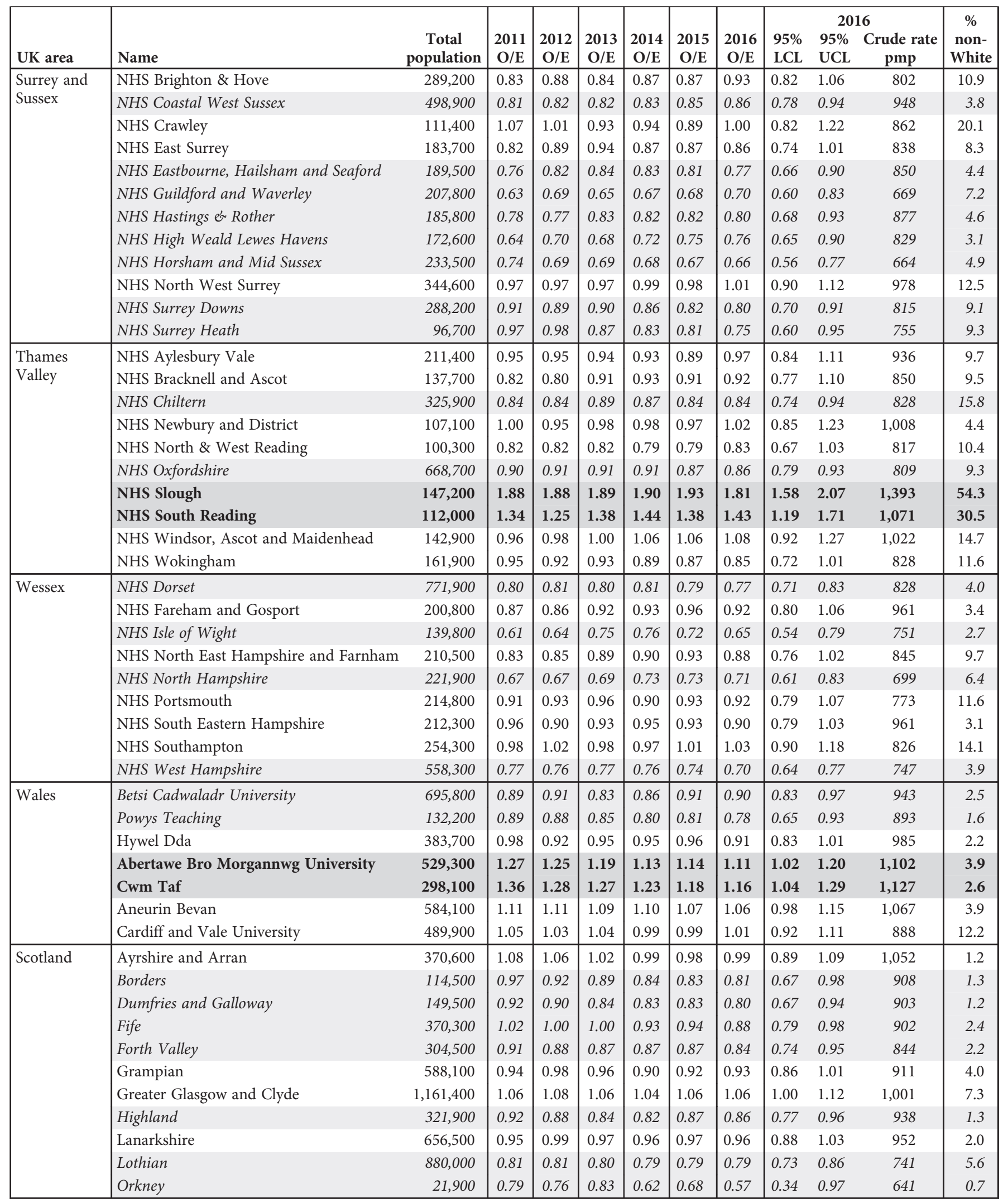


Table 2.5. Continued

\begin{tabular}{|c|c|c|c|c|c|c|c|c|c|c|c|c|}
\hline \multirow[b]{2}{*}{ UK area } & \multirow[b]{2}{*}{ Name } & \multirow[b]{2}{*}{$\begin{array}{c}\text { Total } \\
\text { population }\end{array}$} & \multirow[b]{2}{*}{$\begin{array}{l}2011 \\
\mathrm{O} / \mathrm{E}\end{array}$} & \multirow[b]{2}{*}{$\begin{array}{r}2012 \\
\mathrm{O} / \mathrm{E} \\
\end{array}$} & \multirow[b]{2}{*}{$\begin{array}{l}2013 \\
\mathrm{O} / \mathrm{E} \\
\end{array}$} & \multirow[b]{2}{*}{$\begin{array}{c}2014 \\
\mathrm{O} / \mathrm{E} \\
\end{array}$} & \multirow[b]{2}{*}{$\begin{array}{r}2015 \\
\mathrm{O} / \mathrm{E} \\
\end{array}$} & \multirow[b]{2}{*}{$\begin{array}{l}2016 \\
\mathrm{O} / \mathrm{E} \\
\end{array}$} & \multicolumn{3}{|c|}{2016} & \multirow{2}{*}{$\begin{array}{c}\begin{array}{c}\% \\
\text { non- } \\
\text { White }\end{array} \\
\end{array}$} \\
\hline & & & & & & & & & $\begin{array}{l}95 \% \\
\text { LCL }\end{array}$ & $\begin{array}{l}95 \% \\
\text { UCL }\end{array}$ & $\begin{array}{c}\text { Crude rate } \\
\text { pmp }\end{array}$ & \\
\hline \multirow{3}{*}{$\begin{array}{l}\text { Scotland } \\
\text { (cont.) }\end{array}$} & Shetland & 23,200 & 0.50 & 0.49 & 0.51 & 0.50 & 0.65 & 0.67 & 0.41 & 1.09 & 690 & 1.5 \\
\hline & Tayside & 415,500 & 1.04 & 0.99 & 0.95 & 0.93 & 0.95 & 0.92 & 0.84 & 1.02 & 948 & 3.2 \\
\hline & Western Isles & 26,900 & 0.67 & 0.57 & 0.55 & 0.70 & 0.81 & 0.82 & 0.55 & 1.21 & 929 & 0.9 \\
\hline \multirow{5}{*}{$\begin{array}{l}\text { Northern } \\
\text { Ireland }\end{array}$} & Belfast & 354,700 & 1.15 & 1.17 & 1.16 & 1.15 & 1.13 & 1.16 & 1.05 & 1.29 & 1,023 & 3.2 \\
\hline & Northern & 473,100 & 1.04 & 1.03 & 1.02 & 1.02 & 1.00 & 0.97 & 0.89 & 1.07 & 920 & 1.2 \\
\hline & Southern & 377,200 & 1.01 & 0.96 & 0.97 & 0.98 & 1.01 & 1.04 & 0.94 & 1.16 & 912 & 1.2 \\
\hline & South Eastern & 356,700 & 0.90 & 0.88 & 0.86 & 0.83 & 0.89 & 0.92 & 0.82 & 1.03 & 889 & 1.3 \\
\hline & Western & 300,400 & 1.08 & 0.99 & 0.97 & 1.04 & 1.08 & 1.11 & 1.00 & 1.25 & 1,012 & 1.0 \\
\hline
\end{tabular}

Note that there was a merger between South Manchester CCG, North Manchester CCG and Central Manchester CCG into a single Manchester CCG. Due to boundary changes, a new Morecambe Bay CCG was created covering Lancashire North CCG and North Cumbria CCG was reconfigured; here the new CCGs are used

${ }^{a}$ Excluded from the rate analysis for the 2015-2016 period because $15-100 \%$ of their population was covered by Cambridge (based on estimates using 2014 prevalent data)

${ }^{\mathrm{b}}$ Five further CCGs are flagged because between $5-15 \%$ of their population was estimated to be covered by Cambridge and therefore prevalence ratios for 2015 and 2016 are likely underestimated

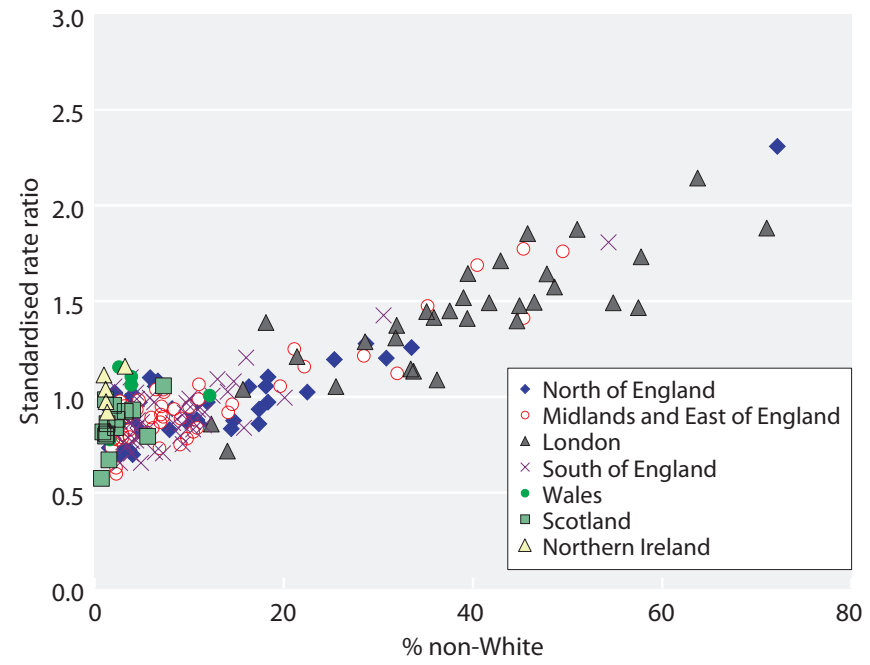

Table 2.7. Median time on RRT of prevalent patients on $31 / 12 / 2016$

\begin{tabular}{lrc}
\hline Modality & $N$ & $\begin{array}{c}\text { Median time treated } \\
\text { (years) }\end{array}$ \\
\hline Haemodialysis & 24,443 & 3.2 \\
Peritoneal dialysis & 3,563 & 1.5 \\
Transplant & 31,836 & 10.3 \\
All RRT & $\mathbf{5 9 , 8 4 2}$ & $\mathbf{6 . 2}$ \\
\hline
\end{tabular}

For patients who recovered for $>90$ days and then subsequently restarted RRT the median time from the start of RRT was calculated from the most recent start date

Patients with an initial treatment modality of transferred in or transferred out were excluded from the calculation of median time on RRT since their treatment start date was not accurately known

Fig. 2.3. SPRs for CCG/HB areas by percentage non-White on $31 / 12 / 2016$ (excluding areas with $<5 \%$ ethnic minorities)

Table 2.6. SPRs of RRT for each region in England and for Wales, Scotland and Northern Ireland in 2016

\begin{tabular}{|c|c|c|c|c|c|}
\hline UK area & Total population & $\mathrm{O} / \mathrm{E}$ & $95 \% \mathrm{LCL}$ & 95\% UCL & Crude rate pmp \\
\hline Midlands and East of England* & $13,792,915$ & 0.99 & 0.98 & 1.01 & 960.9 \\
\hline South England & $14,271,741$ & 0.88 & 0.87 & 0.90 & 882.6 \\
\hline Wales & $3,113,150$ & 1.00 & 0.96 & 1.03 & $1,005.1$ \\
\hline Scotland & $5,404,700$ & 0.92 & 0.89 & 0.94 & 915.1 \\
\hline
\end{tabular}

$\mathrm{O} / \mathrm{E}$ - observed/expected SPR given the age/sex breakdown of each region

Bold - higher than expected SPR

*Eight CCGs covered by Cambridge (NHS Cambridgeshire and Peterborough, NHS South Norfolk, NHS West Norkfolk, NHS West Suffolk, NHS Mid Essex, NHS North East Essex, NHS West Essex, NHS Bedfordshire) were excluded from the rate analysis for the 2015-2016 period because $15-100 \%$ of their population was covered by Cambridge, based on estimates using 2014 prevalent data 
Table 2.8. Median age of prevalent RRT patients by treatment modality in renal centres on $31 / 12 / 2016$

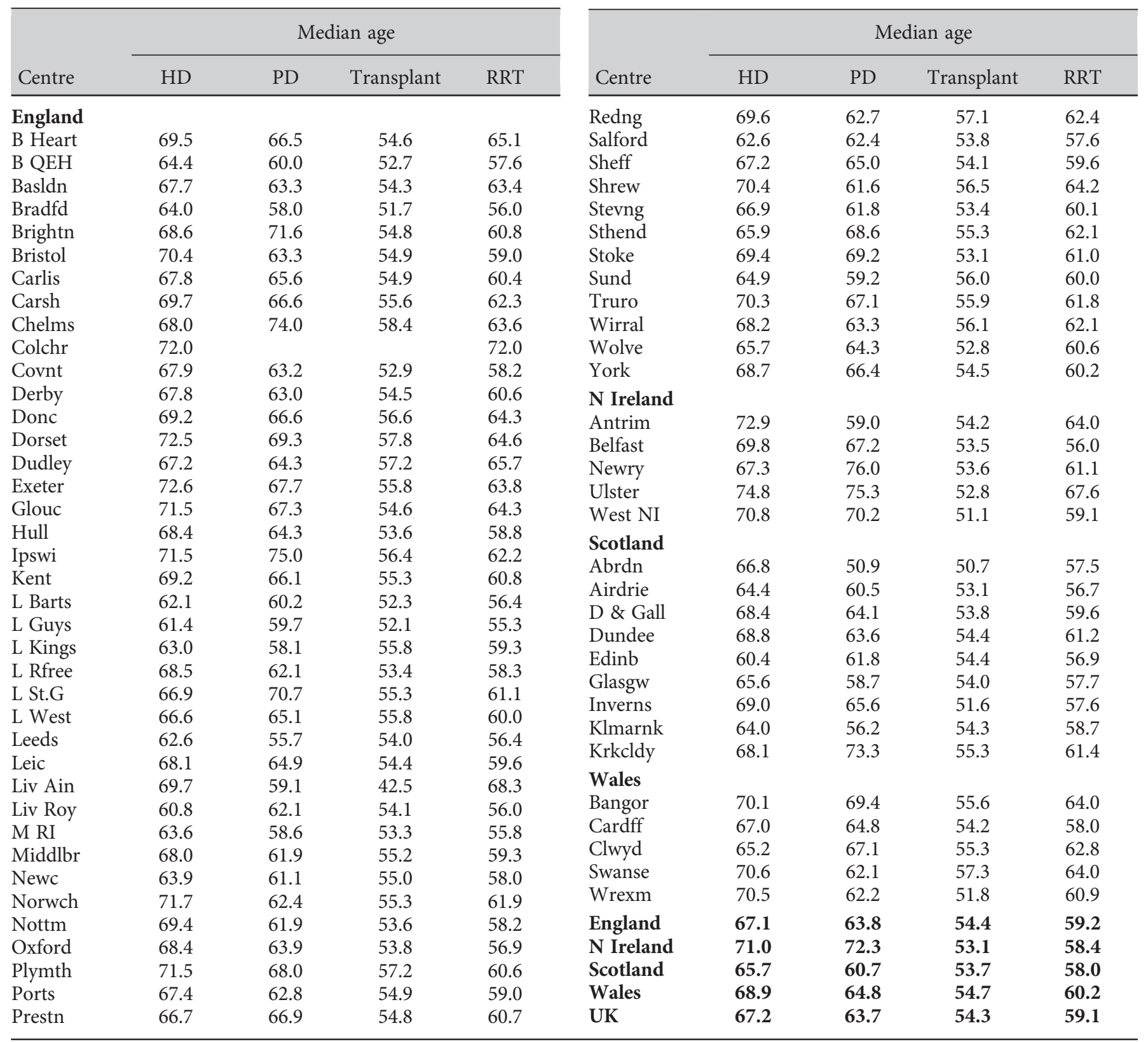

Blank cells indicate no patients on that treatment modality attending that centre when data were collected

UK RRT prevalent patients by using the age and sex distribution of the UK population by CCG/HB (from mid2016 population estimates), allowed estimation of crude prevalence by age and sex (figure 2.5). This shows a progressive increase in prevalence with age, peaking at $2,276 \mathrm{pmp}$ (similar to the 2,270 pmp estimate in 2015) in the age group 75-79 years then a rapid decline thereafter. Crude RRT prevalence in males exceeded that of females for all age groups. The difference was smallest in younger patients and was greatest from the age of 70 years onwards. RRT prevalence in males was highest in the 80-84 years group $(3,072 \mathrm{pmp})$ and for females it was in the 70-74 years group (1,657 pmp). Survival on RRT by sex is described in chapter 5 .

\section{Ethnicity}

Key to understanding differences in RRT prevalence between regions is understanding the ethnic diversity of 
Table 2.9. Percentage of prevalent RRT patients in each age group by centre on $31 / 12 / 2016$

\begin{tabular}{|c|c|c|c|c|c|}
\hline Centre & $N$ & $18-39$ years & $40-64$ years & $65-74$ years & $75+$ years \\
\hline B Heart & 654 & 9.3 & 40.5 & 21.9 & 28.3 \\
\hline B QEH & 2,394 & 15.7 & 51.6 & 19.0 & 13.7 \\
\hline Basldn & 276 & 12.0 & 40.9 & 24.6 & 22.5 \\
\hline Brightn & 996 & 11.9 & 45.5 & 22.6 & 20.0 \\
\hline Bristol & 1,470 & 14.6 & 48.4 & 20.7 & 16.3 \\
\hline Carlis & 279 & 13.3 & 48.4 & 19.4 & 19.0 \\
\hline Carsh & 1,641 & 8.3 & 46.6 & 22.9 & 22.2 \\
\hline Chelms & 278 & 11.2 & 41.4 & 25.9 & 21.6 \\
\hline Colchr & 124 & 6.5 & 25.0 & 27.4 & 41.1 \\
\hline Dudley & 346 & 8.4 & 40.8 & 26.9 & 24.0 \\
\hline Exeter & 1,017 & 10.2 & 42.3 & 24.8 & 22.7 \\
\hline Glouc & 470 & 8.5 & 42.6 & 23.4 & 25.5 \\
\hline Hull & 858 & 14.2 & 49.2 & 21.3 & 15.3 \\
\hline Ipswi & 411 & 8.8 & 47.0 & 23.1 & 21.2 \\
\hline Kent & 1,070 & 11.9 & 46.7 & 24.3 & 17.1 \\
\hline L Barts & 2,372 & 15.1 & 56.7 & 17.6 & 10.6 \\
\hline L Guys & 2,098 & 18.7 & 54.8 & 16.9 & 9.6 \\
\hline L Kings & 1,108 & 8.6 & 55.3 & 18.9 & 17.2 \\
\hline L Rfree & 2,177 & 15.6 & 49.5 & 18.4 & 16.4 \\
\hline L St.G & 863 & 11.7 & 47.9 & 24.0 & 16.5 \\
\hline Newc & 1,053 & 15.2 & 52.4 & 20.7 & 11.7 \\
\hline Norwch & 774 & 11.2 & 45.0 & 24.5 & 19.3 \\
\hline Nottm & 1,152 & 15.9 & 49.7 & 17.9 & 16.6 \\
\hline Oxford & 1,767 & 14.2 & 53.3 & 19.7 & 12.8 \\
\hline Plymth & 513 & 12.1 & 48.5 & 23.6 & 15.8 \\
\hline Ports & 1,693 & 12.8 & 50.5 & 21.0 & 15.7 \\
\hline Prestn & 1,206 & 10.8 & 49.8 & 25.1 & 14.3 \\
\hline Redng & 794 & 8.6 & 48.9 & 23.2 & 19.4 \\
\hline Salford & 1,022 & 13.1 & 53.4 & 20.5 & 13.0 \\
\hline Sheff & 1,427 & 14.4 & 48.6 & 20.5 & 16.5 \\
\hline Shrew & 375 & 9.6 & 41.9 & 24.8 & 23.7 \\
\hline Stevng & 904 & 11.8 & 48.8 & 18.9 & 20.5 \\
\hline Sthend & 237 & 10.5 & 45.6 & 20.7 & 23.2 \\
\hline Stoke & 827 & 12.9 & 45.2 & 22.0 & 19.8 \\
\hline Sund & 507 & 10.8 & 50.7 & 25.2 & 13.2 \\
\hline Truro & 428 & 10.7 & 46.3 & 23.4 & 19.6 \\
\hline Wirral & 337 & 11.3 & 43.6 & 23.7 & 21.4 \\
\hline Wolve & 569 & 11.6 & 48.3 & 20.4 & 19.7 \\
\hline York & 535 & 13.6 & 46.7 & 21.3 & 18.3 \\
\hline
\end{tabular}


Table 2.9. Continued

\begin{tabular}{|c|c|c|c|c|c|}
\hline \multirow[b]{2}{*}{ Centre } & \multirow[b]{2}{*}{$N$} & \multicolumn{4}{|c|}{ Percentage of patients } \\
\hline & & $18-39$ years & 40-64 years & $65-74$ years & $75+$ years \\
\hline Antrim & 241 & 9.1 & 45.2 & 22.0 & 23.7 \\
\hline Belfast & 829 & 16.5 & 53.9 & 16.9 & 12.7 \\
\hline Newry & 237 & 13.1 & 47.7 & 19.4 & 19.8 \\
\hline \multicolumn{6}{|l|}{ Scotland } \\
\hline Abrdn & 557 & 17.8 & 50.1 & 21.4 & 10.8 \\
\hline Airdrie & 440 & 16.4 & 51.1 & 18.2 & 14.3 \\
\hline D \& Gall & 131 & 9.2 & 47.3 & 26.7 & 16.8 \\
\hline Dundee & 420 & 7.6 & 51.2 & 22.6 & 18.6 \\
\hline Edinb & 780 & 14.2 & 57.7 & 18.8 & 9.2 \\
\hline \multicolumn{6}{|l|}{ Wales } \\
\hline Bangor & 180 & 11.1 & 41.1 & 27.8 & 20.0 \\
\hline Cardff & 1,630 & 13.5 & 52.0 & 21.2 & 13.3 \\
\hline Clwyd & 178 & 12.4 & 43.8 & 24.7 & 19.1 \\
\hline Swanse & 768 & 10.4 & 41.7 & 23.2 & 24.7 \\
\hline Wrexm & 310 & 13.2 & 44.8 & 19.4 & 22.6 \\
\hline England & 51,810 & 13.2 & 49.8 & 20.9 & 16.1 \\
\hline N Ireland & 1,780 & 13.7 & 48.6 & 19.4 & 18.4 \\
\hline Scotland & 4,955 & 13.5 & 53.8 & 20.2 & 12.5 \\
\hline Wales & 3,066 & 12.5 & 47.6 & 22.1 & 17.8 \\
\hline UK & 61,611 & 13.2 & 49.9 & 20.9 & 16.0 \\
\hline
\end{tabular}

the patient groups. As such, the completeness of ethnicity data provided by renal centres is important. As in 2015, 61 of the 70 centres (87.1\%) providing patient-level data provided ethnicity data that were at least $90 \%$ complete (table 2.10 ), an improvement on only 36 centres

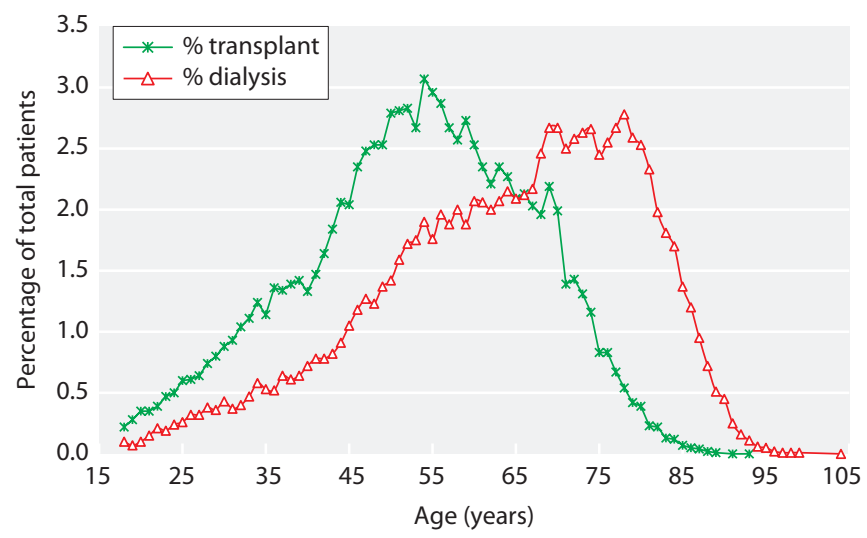

Fig. 2.4. Age profile of prevalent RRT patients by modality on $31 / 12 / 2016$

UK RRT prevalence in 2016 in 2006. Overall ethnicity completeness for prevalent RRT patients has reached a stable $93.6 \%$ for the UK in 2016 compared to $93.3 \%$ in 2015. Data completeness was very high in England, Wales and Northern Ireland (98.7\%, 99.5\% and 98.3\%, respectively), but much lower in Scotland (35.1\%). Completeness in Scotland is

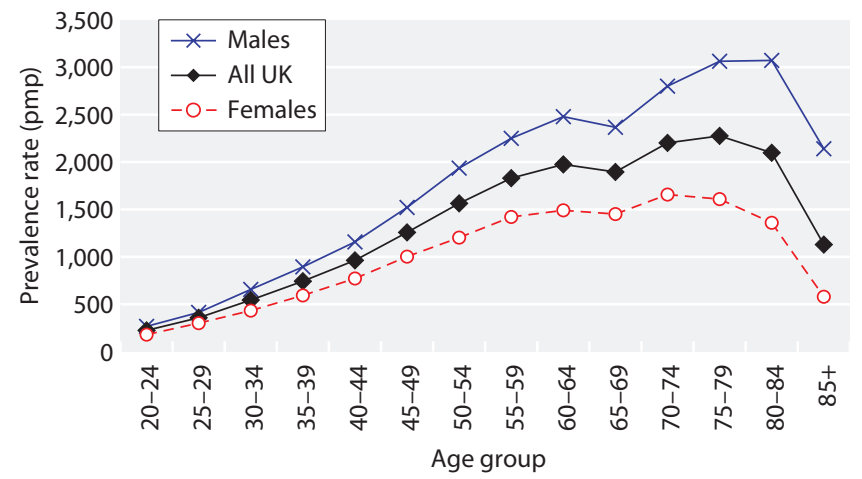

Fig. 2.5. Prevalence of RRT patients pmp by age and sex on 31/12/2016

Nephron 2018;139(suppl1):47-74 
Table 2.10. Ethnicity of prevalent RRT patients by centre on $31 / 12 / 2016$

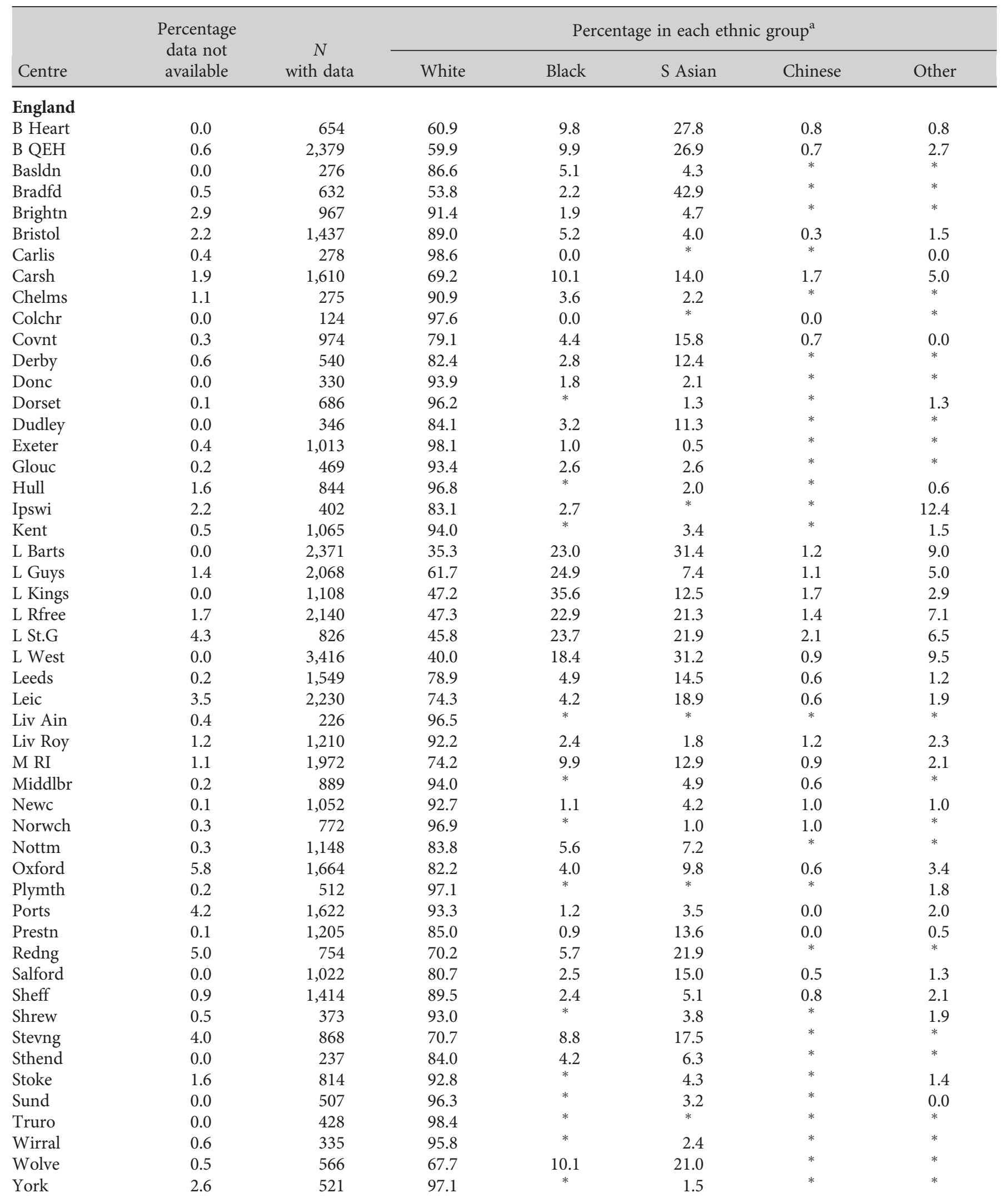


Table 2.10. Continued

\begin{tabular}{|c|c|c|c|c|c|c|c|}
\hline \multirow[b]{2}{*}{ Centre } & \multirow{2}{*}{$\begin{array}{l}\text { Percentage } \\
\text { data not } \\
\text { available }\end{array}$} & \multirow{2}{*}{$\begin{array}{c}N \\
\text { with data }\end{array}$} & \multicolumn{5}{|c|}{ Percentage in each ethnic group ${ }^{a}$} \\
\hline & & & White & Black & S Asian & Chinese & Other \\
\hline \multicolumn{8}{|l|}{ N Ireland } \\
\hline Antrim & 0.0 & 241 & 99.6 & $*$ & 0.0 & $*$ & 0.0 \\
\hline Belfast & 3.7 & 798 & 97.5 & 0.8 & 1.3 & * & $*$ \\
\hline Newry & 0.0 & 237 & 98.3 & $*$ & * & * & 0.0 \\
\hline Ulster & 0.0 & 166 & 96.4 & $*$ & $*$ & * & 0.0 \\
\hline West NI & 0.0 & 307 & 98.7 & $*$ & * & * & 0.0 \\
\hline \multicolumn{8}{|l|}{ Scotland } \\
\hline Abrdn & 60.7 & 219 & & & & & \\
\hline Airdrie & 28.4 & 315 & 95.2 & $*$ & 3.5 & * & * \\
\hline D \& Gall & 75.6 & 32 & & & & & \\
\hline Dundee & 60.5 & 166 & & & & & \\
\hline Edinb & 71.0 & 226 & & & & & \\
\hline Glasgw & 76.7 & 408 & & & & & \\
\hline Inverns & 38.5 & 160 & 95.6 & * & * & 0.0 & * \\
\hline Klmarnk & 56.6 & 138 & & & & & \\
\hline Krkcldy & 74.6 & 75 & & & & & \\
\hline \multicolumn{8}{|l|}{ Wales } \\
\hline Bangor & 1.7 & 177 & 98.3 & * & 0.0 & 0.0 & $*$ \\
\hline Cardff & 0.3 & 1,625 & 92.6 & 0.4 & 5.0 & 0.4 & 1.6 \\
\hline Clwyd & 1.7 & 175 & 97.7 & 0.0 & $*$ & 0.0 & * \\
\hline Swanse & 0.0 & 768 & 97.7 & $*$ & 1.7 & 0.0 & * \\
\hline Wrexm & 1.0 & 307 & 97.7 & $*$ & * & $*$ & * \\
\hline England & 1.3 & 51,120 & 74.4 & 8.5 & 13.3 & 0.7 & 3.1 \\
\hline $\mathrm{N}$ Ireland & 1.7 & 1,749 & 98.0 & 0.7 & 0.9 & $*$ & * \\
\hline Scotland & 64.9 & 1,739 & 82.0 & 6.8 & 8.4 & 2.0 & 0.7 \\
\hline Wales & 0.5 & 3,052 & 95.0 & 0.4 & 3.2 & 0.3 & 1.2 \\
\hline UK & 6.4 & 57,660 & 76.4 & 7.8 & 12.2 & 0.7 & 2.9 \\
\hline
\end{tabular}

${ }^{\mathrm{a}}$ See appendix $\mathrm{H}$ for ethnicity coding

*Values suppressed due to small numbers (primary or secondary suppression)

Blank cells - percentage breakdown not shown for centres with less than $50 \%$ data completeness, but these centres are included in national averages

improving however and only three years ago was $23 \%$. In 2016, completeness of ethnicity data was highest in prevalent transplant patients (42.6\%) which likely reflects improved data recording during the intensive work-up for transplantation.

In $2016,23.6 \%$ of the prevalent UK RRT population (with ethnicity assigned) were from ethnic minorities (25.6\% in England). The proportion of the prevalent UK RRT population (with ethnicity assigned) from ethnic minorities in Wales, Scotland and Northern Ireland was very small, although it should be noted that there was a high level of missing ethnicity data in Scotland as described above. The Office of National Statistics estimates that approximately $13 \%$ of the UK general population is designated as belonging to an ethnic minority [1]. The relative proportion of patients reported to the UKRR as receiving
RRT and belonging to an ethnic minority has increased from $14.9 \%$ in 2007 to $23.6 \%$ in 2016 , which may reflect improvements in coding and reporting of ethnicity data as well as an increasing incidence of ERF and increased referral rates in these populations.

Amongst the centres with more than 50\% returns there was wide variation in the proportion of patients from ethnic minorities, ranging from $0.4 \%$ in Antrim to $64.7 \%$ in London St Bartholomew's.

\section{Primary renal diagnosis}

Primary renal diagnosis (PRD) is associated with patient outcomes and as it could be used for case-mix adjustment, high levels of data completeness are important. Data for PRD were not complete for $3.2 \%$ of patients (table 2.11), but there existed a marked inter-centre 
Table 2.11. PRD in prevalent RRT patients by age and sex on $31 / 12 / 2016$

\begin{tabular}{|c|c|c|c|c|c|c|c|c|}
\hline \multirow[b]{2}{*}{ Primary diagnosis* } & \multirow[b]{2}{*}{$N$} & \multirow{2}{*}{$\begin{array}{c}\% \text { all } \\
\text { patients }\end{array}$} & \multirow{2}{*}{$\begin{array}{c}\text { Intercentre } \\
\text { range } \%\end{array}$} & \multicolumn{2}{|c|}{ Age $<65$} & \multicolumn{2}{|c|}{ Age $\geqslant 65$} & \multirow{2}{*}{$\begin{array}{l}\mathrm{M}: \mathrm{F} \\
\text { ratio }\end{array}$} \\
\hline & & & & $N$ & $\%$ & $N$ & $\%$ & \\
\hline Aetiology uncertain & 9,274 & 15.1 & $4.3-32.9$ & 5,311 & 13.7 & 3,963 & 17.5 & 1.6 \\
\hline Glomerulonephritis & 11,716 & 19.1 & $9.9-26.3$ & 8,367 & 21.5 & 3,349 & 14.8 & 2.1 \\
\hline Pyelonephritis & 6,344 & 10.3 & $4.9-13.6$ & 4,569 & 11.7 & 1,775 & 7.9 & 1.1 \\
\hline Diabetes & 10,375 & 16.9 & $8.7-27.5$ & 6,099 & 15.7 & 4,276 & 18.9 & 1.7 \\
\hline Polycystic kidney & 6,146 & 10.0 & $3.1-16.1$ & 3,935 & 10.1 & 2,211 & 9.8 & 1.1 \\
\hline Hypertension & 3,774 & 6.1 & $1.7-18.1$ & 2,076 & 5.3 & 1,698 & 7.5 & 2.5 \\
\hline Renal vascular disease & 1,809 & 2.9 & $0.5-10.3$ & 396 & 1.0 & 1,413 & 6.3 & 2.0 \\
\hline Other & 10,114 & 16.5 & $11.0-29.4$ & 7,036 & 18.1 & 3,078 & 13.6 & 1.3 \\
\hline Not sent & 1,935 & 3.2 & $0.0-29.5$ & 1,099 & 2.8 & 836 & 3.7 & 1.6 \\
\hline
\end{tabular}

* See appendix H: ERA-EDTA coding

Excluded centre: $\geqslant 40 \%$ primary renal diagnosis aetiology uncertain (Colchr)

difference in completeness of data returns. One centre had $\geqslant 40 \%$ PRD data coded as uncertain and has been excluded from the inter-centre analysis and other analyses where PRD is included in the case-mix adjustment (Colchester, 46\% uncertain PRD); the UK and national totals have been appropriately adjusted. The percentage of patients with uncertain aetiology for the remaining 69 centres providing individual-level data ranged between $4.3 \%$ and $32.9 \%$, which is comparable to recent years. No centre had $>30 \%$ missing data in 2016 .

As observed in previous years, glomerulonephritis (GN) is the most common PRD in the 2016 prevalent cohort at $19.1 \%$ (table 2.11). Diabetic nephropathy is the next most common PRD and accounted for 16.9\% of renal disease in prevalent patients on RRT, although it was more common in the $\geqslant 65$ year age group compared to the younger group (18.9\% vs $15.7 \%)$. The distribution of individual PRDs varied with age; patients aged 65 years and younger were more likely to have GN $(21.5 \%)$ or diabetes $(15.7 \%)$ and less likely to have renal vascular disease $(1.0 \%)$ as the cause of their renal failure. This contrasts with older patients ( $\geqslant 65$ years) among whom $6.3 \%$ had renal vascular disease as the cause of their renal failure. Uncertain aetiology was a more common cause in this age group than amongst younger patients (18.1\% compared with $13.6 \%$ amongst patients $<65$ years).

As described in previous years, the male: female ratio was greater than $1: 1$ for all PRDs (table 2.11). The biggest differences between males and females were for GN (male: female ratio of 2.1), hypertension (2.5) and renal vascular disease (2.0).

Older and younger patients had markedly different trends in the transplant: dialysis ratio by PRD. In individuals aged less than 65 years, the renal transplantation to dialysis ratio was greater than 1 in all PRD groups except diabetic nephropathy and renal vascular disease. In those aged $\geqslant 65$ years, dialysis was more prevalent than renal transplantation in all PRD groups except GN and polycystic kidney disease (PKD) (table 2.12).

\section{Diabetes}

Throughout this section the term 'diabetic nephropathy' is used to denote patients in whom diabetes mellitus is considered to be the primary cause of the kidney disease rather than merely an associated comorbidity. It includes all prevalent patients with type 1 or type 2 diabetes as the PRD (ERA-EDTA coding). This analysis did not differentiate between type 1 and type 2 diabetes

Table 2.12. Transplant: dialysis ratios by age and PRD in the prevalent RRT population on 31/12/2016

\begin{tabular}{lcc}
\hline & \multicolumn{2}{c}{ Transplant : dialysis ratio } \\
\cline { 2 - 3 } Primary diagnosis $^{*}$ & $<65$ years & $\geqslant 65$ years \\
\hline Aetiology uncertain & 2.2 & 0.4 \\
Glomerulonephritis & 2.5 & 1.0 \\
Pyelonephritis & 3.0 & 0.6 \\
Diabetes & 0.9 & 0.2 \\
Polycystic kidney & 3.4 & 2.0 \\
Hypertension & 1.5 & 0.4 \\
Renal vascular disease & 0.9 & 0.1 \\
Other & 2.2 & 0.5 \\
Not sent & 0.8 & 0.1 \\
\hline
\end{tabular}

* Appendix H ERA-EDTA coding

Excluded centre: $\geqslant 40 \%$ primary renal diagnosis aetiology uncertain (Colchr) 
Table 2.13. Age relationships in patients with diabetes and patients without diabetes and modality in prevalent RRT patients on $31 / 12 / 2016$

\begin{tabular}{lcc}
\hline & $\begin{array}{c}\text { Patients with }^{\text {diabetes }}{ }^{\mathrm{a}} \\
\text { d }\end{array}$ & $\begin{array}{c}\text { Patients without } \\
\text { diabetes }^{\mathrm{b}}\end{array}$ \\
\hline : F ratio & 10,375 & 49,177 \\
Median age on 31/12/16 & 1.66 & 1.54 \\
Median age at start of RRT & 62 & 58 \\
Median years on RRT & 56 & 47 \\
\% HD & 3.6 & 7.5 \\
\% PD & 58 & 36 \\
\% transplant & 8 & 5 \\
\hline
\end{tabular}

Excluded centre: $\geqslant 40 \%$ PRD aetiology uncertain (Colchr)

${ }^{a}$ Patients with diabetes: patients with a PRD code of diabetes

${ }^{\mathrm{b}}$ Patients without diabetes: all patients excluding patients with diabetes as a PRD and patients with a missing PRD code

${ }^{\mathrm{c}}$ Median age at start of RRT was calculated from the most recent RRT start date

${ }^{\mathrm{d}}$ Patients with an initial treatment modality of transferred in or transferred out were excluded from the calculation of median age at start of RRT and median years on RRT, since their treatment start date was not accurately known

as this distinction was not made in the data submitted by most centres.

The number of prevalent patients with diabetic nephropathy has increased steadily over the last number of years and grew by $4.7 \%$ to 10,375 in 2016 , from 9,913 in 2015 , representing $17.4 \%$ of all prevalent patients (compared with $13.5 \%$ in 2006) (table 2.13). Men were 1.66 times more likely to have diabetic nephropathy than women. The median age at start of RRT for patients with diabetic nephropathy (56 years) was nine years higher than those with other PRDs (47 years), although the median age at the end of 2016 for prevalent patients with diabetic nephropathy was only four years higher than for individuals without diabetic nephropathy. This reflects reduced survival for patients with diabetes compared with patients without diabetes on RRT. This is also supported by the lower median time on RRT for patients with diabetic nephropathy (3.6 years vs 7.5 years for those without diabetic nephropathy) and this difference in survival has not changed over the last five years (3.4 years in 2016 vs 6.5 years in 2011). The age at starting RRT in those with diabetic nephropathy was four years younger in Scotland compared with the UK average (data not shown).

Patients with diabetic nephropathy had a different distribution of RRT modalities than those without diabetes. Fifty eight percent of patients with diabetic nephropathy were undergoing HD compared with just $36 \%$ of patients
Table 2.14. Treatment modalities by age and diabetes status on $31 / 12 / 2016$

\begin{tabular}{lccccc}
\hline & \multicolumn{2}{c}{$<65$} & & \multicolumn{2}{c}{$\geqslant 65$} \\
\cline { 2 - 3 } \cline { 5 - 6 } & Diabetes $^{\mathrm{a}}$ & \begin{tabular}{c} 
All other $^{\mathrm{c}}$ causes $^{\mathrm{b}}$ \\
\cline { 5 - 6 }$N$
\end{tabular} & & Diabetes $^{\mathrm{a}}$ & $\begin{array}{c}\text { All other } \\
\text { causes }^{\mathrm{b}}\end{array}$ \\
\hline \% HD & 6,099 & 31,690 & & 4,276 & 17,487 \\
\% PD & 44.8 & 25.1 & & 76.8 & 54.7 \\
\% transplant & 7.4 & 4.2 & & 8.2 & 7.2 \\
\hline
\end{tabular}

Excluded centre with $\geqslant 40 \%$ PRD aetiology uncertain (Colchr) ${ }^{a}$ Patients with diabetes are patients with a PRD code of diabetes

${ }^{b}$ Patients without diabetes are calculated as all patients excluding patients with diabetes as a PRD and patients with a missing PRD code

with any other PRD (table 2.13). The percentage of patients with a functioning transplant was much lower in prevalent patients with diabetic nephropathy than in prevalent patients without ( $34 \%$ vs $59 \%$ ). The proportion of patients with diabetic nephropathy with a functioning transplant has increased however since 2006 when only $27 \%$ of patients with diabetic nephropathy had a functioning transplant. For older patients with diabetic nephropathy (age $\geqslant 65$ years), only $15.1 \%$ had a functioning transplant compared with $47.8 \%$ of their peers with a transplant aged under 65 years (table 2.14). Amongst those patients receiving dialysis, a higher proportion of prevalent patients without diabetic nephropathy (18.1\%) were on home dialysis therapies (home HD and PD) compared with prevalent patients with diabetic nephropathy (14.1\%). Both of these trends (those with diabetic nephropathy being more likely to be doing home dialysis than those with other PRDs and less likely to be transplanted) were consistent across all age groups (18-39 years, 40-64 years, 65-74 years, $75+$ years), although as expected the greatest proportion transplanted in both groups are those aged 18-39 years (data not shown).

\section{Modalities of treatment}

Transplantation was the most common treatment modality (53.9\%) for prevalent RRT patients in 2016, followed by centre-based HD (38.3\%) in either hospital centre (17.5\%) or satellite unit (20.8\%) (figure 2.6). Satellite $\mathrm{HD}$ was again more prevalent than in-centre $\mathrm{HD}$, a trend first noted in 2012. Home therapies made up the remaining $7.9 \%$ of treatment therapies, largely $\mathrm{PD}$ in its different formats (5.9\%) which has followed a similar pattern since 2012. The proportion on continuous ambulatory PD (CAPD) and automated PD (APD) was $2.4 \%$ and $3.5 \%$ respectively, although the proportion on APD 


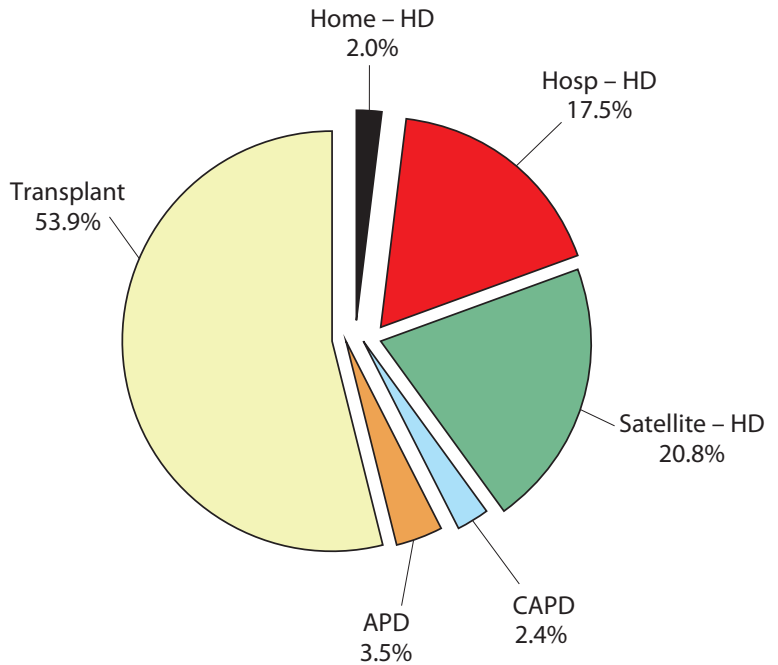

Fig. 2.6. Treatment modality in prevalent RRT patients on $31 / 12 / 2016$

may be an underestimate due to centre level coding issues which mean the UKRR cannot always distinguish between these therapies.

As described earlier, treatment modality was related to patient age. Younger patients (age $<65$ years), were more likely to have a functioning transplant $(66.3 \%)$ when compared with patients aged 65 years and over (32.6\%) (table 2.15). HD was the principal modality in older patients (59.9\%).

Figure 2.7 shows the distribution of RRT modalities by age group. From the age of 45 years onwards, transplant prevalence declined as HD prevalence increased. The proportion of each age group treated by $\mathrm{PD}$ remained relatively stable.

The proportion of prevalent dialysis patients receiving HD varied between centres, ranging from $72.9 \%$ in Carlisle to $100 \%$ in Colchester (table 2.16).

Of the dialysis population, $45.1 \%$ received their treatment at a satellite HD unit in 2016. This figure remained comparable to recent years, but represented an increase from $39.9 \%$ in 2010 . In 2016, the number of centres that had more than $50 \%$ of their HD activity taking place in satellite units was 27 (figure 2.8). Although there were satellite units in Scotland, the data provided for 2016 did not distinguish between main centre and satellite unit HD. As such, it is difficult to accurately assess access to satellite $\mathrm{HD}$ across the UK as a whole so statistics pool only England, Wales and Northern Ireland.

Table 2.15. Percentage of prevalent RRT patients by age group and modality by UK country on $31 / 12 / 2016$

\begin{tabular}{|c|c|c|c|c|c|c|c|c|}
\hline \multirow[b]{2}{*}{ UK country } & \multicolumn{4}{|c|}{$<65$ years } & \multicolumn{4}{|c|}{$\geqslant 65$ years } \\
\hline & $N$ & $\% \mathrm{HD}$ & $\% \mathrm{PD}$ & $\%$ transplant & $N$ & $\% \mathrm{HD}$ & $\% \mathrm{PD}$ & $\%$ transplant \\
\hline England & 32,644 & 29.5 & 4.9 & 65.6 & 19,166 & 60.0 & 7.7 & 32.3 \\
\hline N Ireland & 1,108 & 20.1 & 2.4 & 77.4 & 672 & 61.2 & 7.4 & 31.4 \\
\hline Scotland & 3,333 & 27.5 & 4.2 & 68.4 & 1,622 & 61.0 & 5.5 & 33.4 \\
\hline Wales & 1,842 & 26.2 & 5.6 & 68.1 & 1,224 & 55.5 & 8.3 & 36.2 \\
\hline UK & 38,927 & 28.9 & 4.8 & 66.3 & 22,684 & 59.9 & 7.5 & 32.6 \\
\hline
\end{tabular}

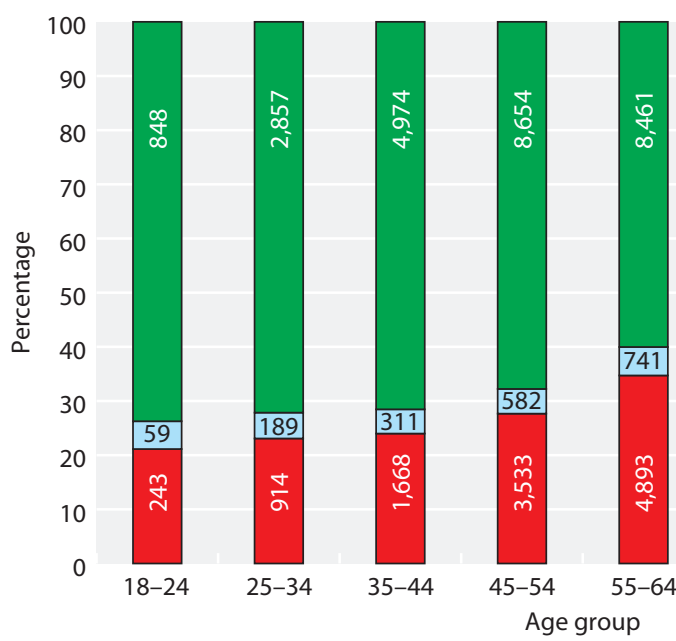

68

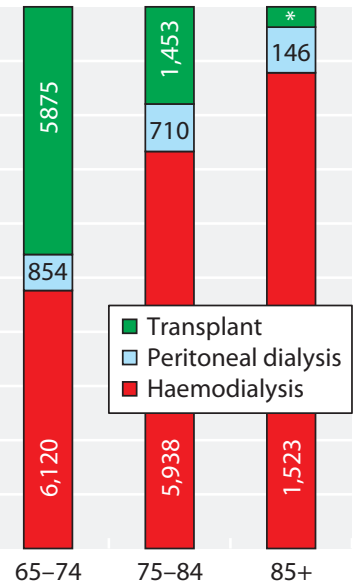

Fig. 2.7. Treatment modality distribution by age in prevalent RRT patients on $31 / 12 / 2016$ ${ }^{*} N=65$ 
Table 2.16 Percentage of prevalent dialysis patients by dialysis modality and centre on 31/12/2016

\begin{tabular}{|c|c|c|c|c|c|c|c|}
\hline \multirow[b]{2}{*}{ Centre } & \multirow[b]{2}{*}{$N$} & \multicolumn{4}{|c|}{$\% \mathrm{HD}$} & \multicolumn{2}{|c|}{$\% \mathrm{PD}$} \\
\hline & & Total & Home & Hospital & Satellite & CAPD & APD \\
\hline \multicolumn{8}{|l|}{ England } \\
\hline B Heart & 483 & 81.8 & 3.9 & 72.9 & 5.0 & 5.6 & 12.6 \\
\hline B QEH & 1,152 & 87.6 & 4.9 & 10.8 & 72.0 & 3.9 & 8.5 \\
\hline Basldn & 196 & 82.7 & $*$ & 64.3 & 17.9 & $*$ & 10.7 \\
\hline Brightn & 524 & 87.6 & 7.1 & 37.8 & 42.8 & 7.6 & 4.8 \\
\hline Bristol & 563 & 90.6 & 3.4 & 16.3 & 70.9 & 3.9 & 5.5 \\
\hline Carlis & 129 & 72.9 & $*$ & 51.9 & 20.9 & $*$ & 24.0 \\
\hline Carsh & 961 & 88.3 & 3.0 & 18.6 & 66.6 & 2.8 & 9.0 \\
\hline Chelms & 166 & 80.1 & $*$ & 78.9 & $*$ & 8.4 & 10.8 \\
\hline Colchr & 124 & 100.0 & $*$ & 100.0 & $*$ & 0.0 & 0.0 \\
\hline Dudley & 253 & 80.2 & 5.5 & 30.0 & 44.7 & 13.8 & 5.5 \\
\hline Exeter & 539 & 84.4 & 1.7 & 8.5 & 74.2 & 6.3 & 9.3 \\
\hline Glouc & 286 & 85.3 & 3.2 & 59.1 & 23.1 & 3.5 & 11.2 \\
\hline Hull & 401 & 82.0 & $*$ & 42.6 & 38.4 & 11.7 & $*$ \\
\hline Ipswi & 182 & 80.2 & 0.0 & 68.7 & 11.5 & 8.2 & 11.5 \\
\hline Kent & 486 & 88.5 & 4.5 & 33.3 & 50.6 & 8.4 & 3.1 \\
\hline L Barts & 1,232 & 83.6 & 1.9 & 36.3 & 45.5 & 1.5 & 14.9 \\
\hline L Guys & 732 & 94.7 & 6.6 & 18.3 & 69.8 & 1.9 & 3.4 \\
\hline L Kings & 672 & 86.5 & 2.7 & 17.4 & 66.4 & 5.2 & 8.3 \\
\hline L Rfree & 889 & 82.0 & 2.3 & 3.6 & 76.2 & 5.5 & 12.5 \\
\hline L St.G & 399 & 88.7 & $*$ & 17.0 & 70.7 & $*$ & 7.3 \\
\hline Norwch & 380 & 87.1 & 4.2 & 50.5 & 32.4 & 12.9 & 0.0 \\
\hline Nottm & 475 & 82.7 & 6.1 & 35.4 & 41.3 & 7.2 & 10.1 \\
\hline Oxford & 545 & 82.6 & 3.5 & 31.2 & 47.9 & 6.2 & 11.0 \\
\hline Plymth & 184 & 78.3 & 4.4 & 65.2 & 8.7 & 7.6 & 14.1 \\
\hline Ports & 711 & 89.5 & 10.6 & 17.2 & 61.7 & 10.6 & 0.0 \\
\hline Prestn & 604 & 93.4 & 6.8 & 19.9 & 66.7 & 1.3 & 5.3 \\
\hline Redng & 359 & 84.4 & 2.2 & 39.0 & 43.2 & 10.0 & 5.6 \\
\hline Salford & 509 & 79.0 & 5.5 & 20.2 & 53.2 & 8.8 & 12.2 \\
\hline Sheff & 671 & 91.8 & 7.9 & 37.6 & 46.4 & 8.2 & 0.0 \\
\hline Shrew & 244 & 84.0 & 7.8 & 42.6 & 33.6 & 3.3 & 12.7 \\
\hline Stevng & 554 & 96.0 & 4.7 & 42.1 & 49.3 & * & * \\
\hline Sthend & 144 & 79.2 & $*$ & 77.1 & $*$ & 20.8 & 0.0 \\
\hline Stoke & 425 & 81.4 & 8.0 & 47.3 & 26.1 & 2.1 & 9.2 \\
\hline Sund & 268 & 93.7 & 2.2 & 61.6 & 29.9 & 3.7 & 2.6 \\
\hline Truro & 188 & 90.4 & 4.8 & 49.5 & 36.2 & 5.3 & 4.3 \\
\hline Wirral & 221 & 90.0 & 4.5 & 41.2 & 44.3 & 2.3 & 7.7 \\
\hline Wolve & 384 & 81.8 & 7.8 & 50.3 & 23.7 & 3.9 & 12.0 \\
\hline York & 231 & 85.7 & 6.1 & 29.0 & 50.7 & 10.0 & 4.3 \\
\hline
\end{tabular}


Table 2.16 Continued

\begin{tabular}{|c|c|c|c|c|c|c|c|}
\hline Centre & $N$ & Total & Home & Hospital & Satellite & CAPD & APD \\
\hline \multicolumn{8}{|l|}{ N Ireland } \\
\hline Antrim & 139 & 88.5 & $*$ & 87.8 & 0.0 & $*$ & 11.5 \\
\hline Belfast & 218 & 89.0 & 4.1 & 84.9 & 0.0 & 0.0 & 11.0 \\
\hline Newry & 108 & 80.6 & $*$ & 77.8 & 0.0 & $*$ & 18.5 \\
\hline \multicolumn{8}{|l|}{ Scotland } \\
\hline Abrdn & 252 & 91.7 & $*$ & 90.1 & $*$ & 4.0 & 4.4 \\
\hline Airdrie & 209 & 88.5 & 0.0 & 88.5 & 0.0 & 3.8 & 7.7 \\
\hline D \& Gall & 60 & 83.3 & $*$ & 78.3 & $*$ & 8.3 & 8.3 \\
\hline Dundee & 200 & 89.5 & * & 88.5 & 0.0 & 9.0 & $*$ \\
\hline Edinb & 326 & 88.7 & $*$ & 86.8 & 0.0 & $*$ & 10.1 \\
\hline \multicolumn{8}{|l|}{ Wales } \\
\hline Bangor & 91 & 82.4 & 11.0 & 50.6 & 20.9 & 5.5 & 12.1 \\
\hline Cardff & 592 & 87.3 & 5.2 & 9.5 & 72.6 & 6.9 & 5.7 \\
\hline Clwyd & 88 & 83.0 & $*$ & 78.4 & $*$ & 8.0 & 9.1 \\
\hline Swanse & 440 & 84.8 & 9.1 & 45.7 & 30.0 & 6.1 & 9.1 \\
\hline Wrexm & 157 & 79.0 & $*$ & 60.5 & 13.4 & $*$ & 20.4 \\
\hline England & 24,211 & 87.3 & 4.5 & 32.3 & 50.5 & 5.4 & 7.2 \\
\hline N Ireland ${ }^{\mathbf{a}}$ & 711 & 89.2 & $*$ & 86.8 & 0.0 & $*$ & 10.4 \\
\hline Scotland ${ }^{\mathrm{b}}$ & 2,134 & 89.3 & 2.5 & 86.8 & 0.0 & 3.3 & 7.5 \\
\hline Wales & 1,368 & 85.0 & 6.8 & 34.1 & 44.0 & 5.9 & 9.1 \\
\hline UK & 28,424 & 87.4 & 4.4 & 37.9 & 45.1 & 5.1 & 7.4 \\
\hline
\end{tabular}

* Values suppressed due to small numbers (primary or secondary suppression)

${ }^{a}$ There were no satellite units in Northern Ireland

${ }^{\mathrm{b}}$ All HD patients in Scotland were shown as receiving treatment at home or in centre as no data was available regarding satellite dialysis

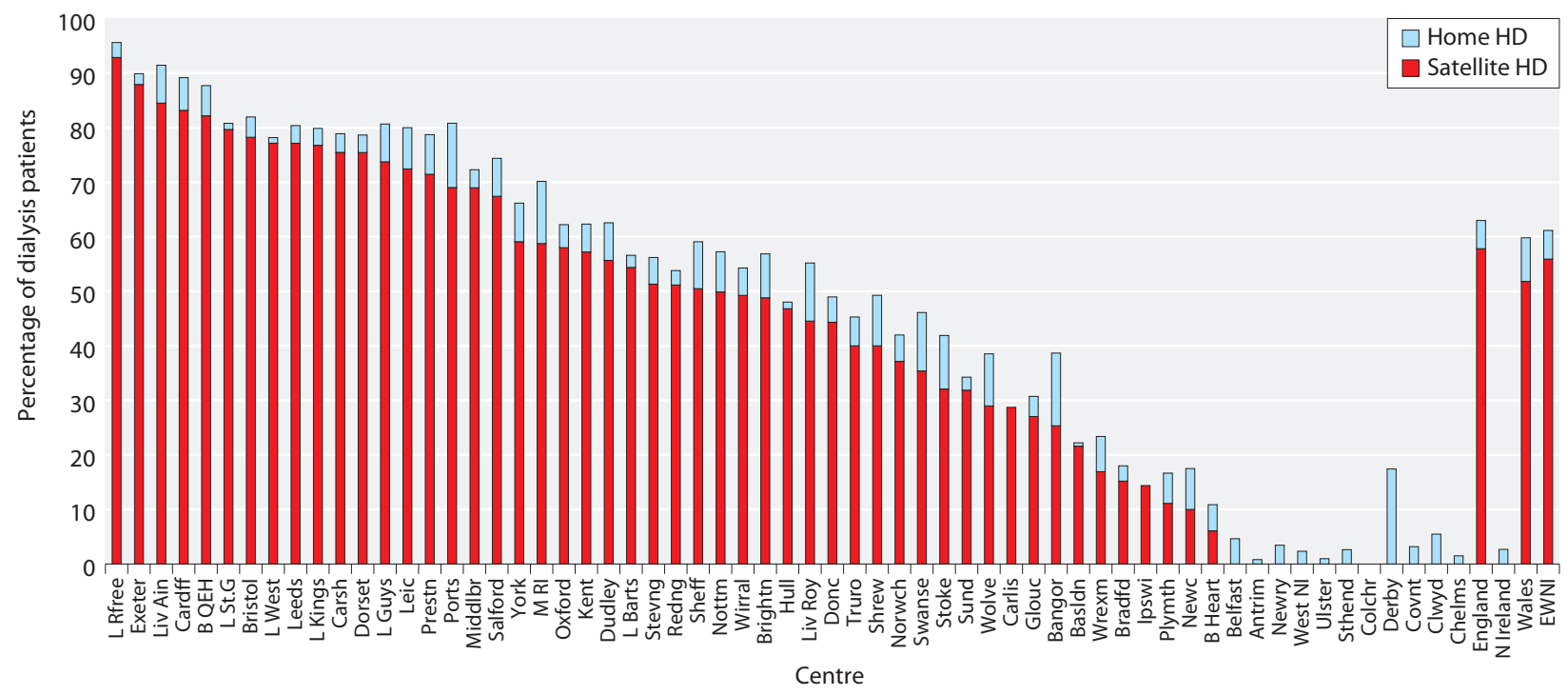

Fig. 2.8. Percentage of prevalent HD patients treated with satellite or home HD by centre on 31/12/2016

${ }^{*}$ Scottish centres excluded as information on satellite HD was not available. No centres in Northern Ireland had satellite dialysis units 


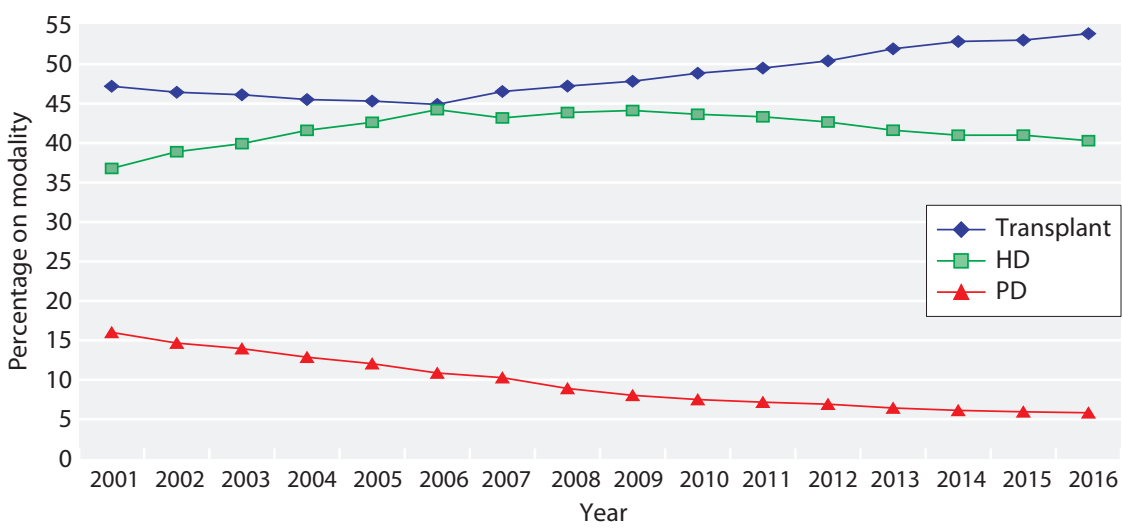

Fig. 2.9. Modality changes in prevalent RRT patients from 2001-2016
Some centres also showed differences in satellite HD provision in 2016 compared to 2015. For example, at London St George's, $70.7 \%$ of patients received dialysis at satellite units in 2016 compared to $49.7 \%$ in 2015. Stevenage had a decrease in the proportion of patients receiving $\mathrm{HD}$ at satellite units from $66.3 \%$ in 2015 to $49.3 \%$ in 2016.

There was also wide variation between centres in the proportion of dialysis patients being managed with APD, ranging from $0.0 \%$ to $24.0 \%$ (table 2.16 ). While in Northern Ireland nearly all PD patients were on APD, across the UK six of the 69 centres with a PD programme did not report having any patients on APD.

\section{Home haemodialysis}

In 2016, the percentage of dialysis patients receiving home $\mathrm{HD}$ varied from $0 \%$ in five centres, to $5 \%$ or greater in 24 centres (table 2.16). In the UK, the overall percentage of dialysis patients receiving home HD has increased from $3.4 \%$ in 2011 to $4.4 \%$ in 2016 .

The proportion of dialysis patients receiving home HD was greatest in Wales at $6.8 \%$, compared with $2.4 \%$ in Northern Ireland, $4.5 \%$ in England and 2.5\% in Scotland (figure 2.8, table 2.16). By comparison, in 2007, the proportion of patients receiving home HD was $2 \%$ in each of the four UK countries. More recently, 30 renal centres across the UK had an increase in the proportion of individuals on home HD compared with 2015.

\section{Change in modality}

The relative proportion of RRT modalities in prevalent patients has changed dramatically over the past 16 years. The main features are depicted in figure 2.9, which describes a year on year decline in the proportion of patients treated using PD since 2001 and a drop of $5.0 \%$ over the last ten years. The absolute number of patients on PD decreased from 4,293 patients in 2006 to 3,589 patients in 2016. Time on PD has decreased over the last nine years, from a median of 2.0 years in 2007 to 1.5 years in 2016 probably reflecting increased transplantation rates in this largely younger patient group and reducing PD technique survival rates. The percentage of patients undergoing PD for more than seven years was only $8.7 \%$.

The proportion of all RRT patients being treated with HD has fallen slightly since 2009 from $44.1 \%$ to $40.3 \%$ although this still represents an increase in absolute numbers on HD (from 21,671 to 24,832) as well as an increase in HD prevalence (from 354 to $385 \mathrm{pmp}$ ).

The proportion of patients with a functioning transplant has been increasing since 2007 (46.5\%) to 53.9\% in 2016. This probably reflects both an increasing number of incident transplants $(2,218$ adults and children in 2007 [2] to 3,174 in 2016) as well as increasing survival of prevalent transplant patients.

Figure 2.10 depicts in more detail the modality changes in the prevalent dialysis population during this time. The data show a clear reduction in patients treated by CAPD over time and an increase in satellite HD coupled with a reduction in hospital HD.

\section{International comparisons}

There were marked differences in RRT prevalence between countries (figure 2.11). RRT prevalence in Northern European countries (including the UK), Australia and New Zealand was lower than in Southern Europe which was lower than the USA, Canada and Japan. Identifying the source of these differences is complicated by differences in healthcare systems, patient registry coverage, approaches to conservative care and incidence rates in these countries. 

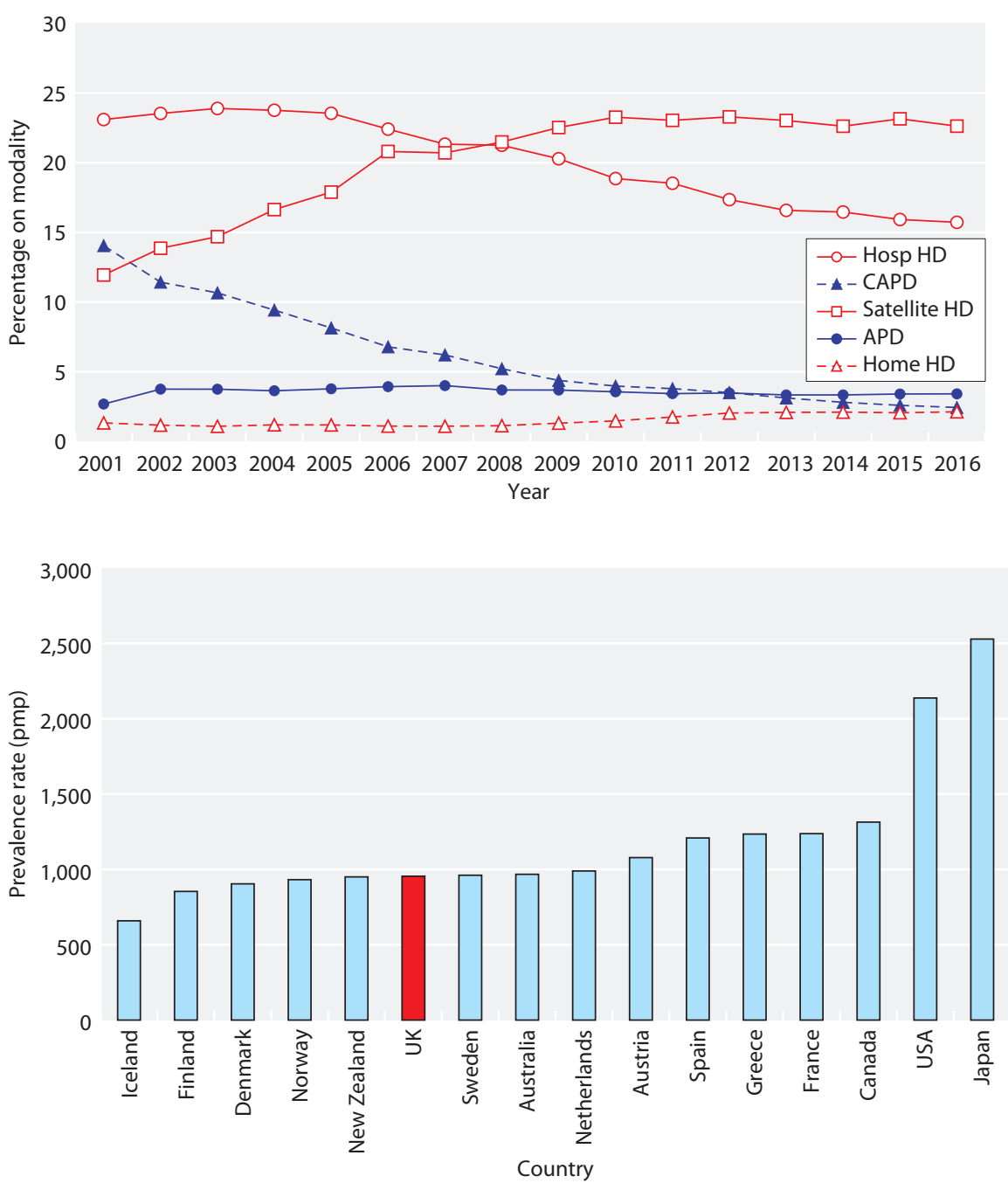

Fig. 2.10. Detailed dialysis modality changes in prevalent RRT patients from 2001-2016 *Scottish centres excluded as information on satellite $\mathrm{HD}$ was not available
Fig. 2.11. RRT prevalence (pmp) by country in 2015

Non-UK data from the USRDS available at https://www.usrds.org/2017/ref/ESRD_Ref_N_ International_2017.xlsx

The UK data include paediatric patients to correspond with the data from the other countries

All rates unadjusted

Data for France include 26 regions (excluding Martinique); data for Canada excludes Quebec

\section{Discussion}

Prevalence of RRT continued to increase in the UK, with an absolute increase in the number of adults receiving RRT of 3\% between 2015 and 2016. The majority of this increase was in people with a functioning renal transplant (5\% increase); with a $1 \%$ increase in the number of people receiving HD. There was significant variation between centres in the change in the number of prevalent RRT patients between 2015 and 2016; one centre experienced a $4 \%$ fall whereas another had a $20 \%$ increase. Whether this variation reflects local differences or recent changes in RRT choices, a one-off movement of patients, historical differences in dialysis planning, or differences in genuine need for RRT would require local interpretation.

The change in prevalence of RRT represents a balance between new patients to RRT (discussed in chapter 1 on incidence), movements between treatment types (discussed particularly in chapter 9 on access to transplantation) and mortality (discussed in chapter 5 on survival). Occasionally it can be artefactual due to a change in reporting practice by centres. The growth in the prevalence of adults with a functioning transplant for example, in part represents the success of recent increases in transplant numbers and a lower mortality rate (compared with those receiving dialysis).

There have been constraints such as historic in-centre HD capacity because of high capital costs, people requiring a renal transplant being limited by the availability of donor organs and people preferring home therapies being limited by access to equipment or training resources. Therefore, it is not possible to conclude from this report whether the prevalence of RRT (in its entirety or by modality) reflects the genuine need for RRT in a particular locality or whether there was (currently unmeasured) unmet need. The UKRR has started collecting 
information about patients with CKD stage 4 and 5 from renal centres which it is hoped will enable a better description of the prevalence of people with CKD5 not on dialysis. This will include those having dialysis preparation, those waiting to start RRT and those having conservative kidney care which will help assess this further in future years.

$\mathrm{PD}$ as a treatment type continued to grow very slowly in absolute numbers and has decreased as a proportion of all those on RRT. The numbers of people treated by home HD continued to increase (an average annual increase of $15 \%$ pmp since 2012) but this was from a low base so represents an increase from 737 patients in 2012 to 1,188 in 2016. Increasing the number of people able to dialyse at home is one of the three priorities identified by the Kidney Quality Improvement Partnership (KQuIP) along with vascular access and transplant first. At regional KQuIP meetings, several local renal teams have identified access to home therapies and renal transplantation as topics that they will work to improve in the coming year. Evaluation of their efforts on these priorities will be collected and published through the UKRR annual report, allowing teams to focus their efforts on the improvement programmes themselves and is a good example of how a national registry can help facilitate local improvement.

The population of the UK continues to age which was also reflected in the population receiving RRT with a median age of 59 years compared with 55 years in the year 2005. Age appears to be one of a group of factors (including diabetes as PRD) which influences the proportions on each RRT modality. Patients with a functioning renal transplant are younger on average (54 years) than those on PD (64 years) and (predominantly incentre) HD (67 years). Whilst age confounds the treatment modality for those with diabetes as the cause of their ERF, at any age the proportion of those with diabetes who have a functioning renal transplant was lower than those who had an alternate cause of ERF.

Chronic kidney disease (CKD) is associated with several conditions which increase in prevalence with age (diabetes, hypertension and cardiovascular disease for example). It is unsuprising therefore that the peak prevalence of RRT pmp was in the 80-84 age group for men and the 70-74 age group for women. The prevalence of CKD stages 3-5 was higher amongst women in the UK either in GP practice populations [3], or health surveys [4] and women in the UK general population have a longer life expectancy than men [5]. Whilst it is thought that women progress to ERF more slowly [6] and once on dialysis lose their general population survival advantage over men [7], the full explanation for why in contrast a greater proportion of people receiving RRT were men is not known. Information obtained from patients in renal centres with CKD 4-5 may help unravel this paradox better in the future.

\section{Acknowledgement}

The (non-UK) data reported in the section on International comparisons have been supplied by the United States Renal Data System (USRDS). The interpretation and reporting of these data are the responsibility of the author(s) and in no way should be seen as an official policy or interpretation of the U.S. government.

Conflicts of interest: the authors declare no conflicts of interest

\section{References}

1 Office of National Statistics. www.statistics.gov.uk

2 Webb L, et al., UK Renal Registry 13th Annual Report: Chapter 3 Demographic and biochemistry profile of kidney transplant recipients in the UK in 2009: national and centre-specific analyses. Nephron Clin Pract. 2011; 119 (suppl 2): c53-84. doi: 10.1159/000331745)

3 Nitsch D, Caplin B, Hull S, Wheeler D. National Chronic Kidney Disease Audit: Report (Part 1) - HQIP [Internet]. Available from: https://www. hqip.org.uk/public/cms/253/625/19/704/08532\%20CKD\%20Audit\%20 Report\%20Jan\%2017\%20FINAL.pdf?realName $=$ HtoEm0.pdf\&v=0

4 Roderick P, Roth M, Mindell J. Prevalence of chronic kidney disease in England: Findings from the 2009 Health Survey for England. Journal of Epidemiology and Community Helath. 2011;65(suppl 2);A12

5 Office of National Statistics. Life expectancies [Internet]. Available from: https://www.ons.gov.uk/peoplepopulationandcommunity/birthsdeathsand marriages/lifeexpectancies

6 Eriksen BO, Ingebretsen OC. The progression of chronic kidney disease: a 10 -year population-based study of the effects of gender and age. Kidney International 2006;69(2):375-82

7 Hecking M, Bieber BA, Ethier J, Kautzky-Willer A, Sunder-Plassman G, Saemann MD et al. Sex-specific differences in haemodialysis prevalence and practices and the male-to-female mortality rate: The Dialysis Outcomes and Practice Patterns Study (DOPPS). PLOS Medicine 2014; https://doi.org/10.1371/journal.pmed.1001750 
